\title{
A generalization of Fiedler's lemma and the spectra of $H$-join of graphs ${ }^{\text {th }}$
}

\author{
M. Saravanan ${ }^{\text {a,* }}$, S.P. Murugan ${ }^{\text {b }}$, G. Arunkumar ${ }^{\mathrm{c}}$ \\ a Government Arts and Science College (Erstwhile Madurai Kamaraj University \\ Constituent College), Sattur, India \\ b Indian Institute of Science Education and Research, Mohali, India \\ c Indian Institute of Science, Bangalore, India
}

\section{A R T I C L E I N F O}

\section{Article history:}

Received 28 September 2020

Accepted 15 April 2021

Available online 26 April 2021

Submitted by R. Brualdi

\section{$M S C$ :}

$05 \mathrm{C} 50$

$05 \mathrm{C} 76$

Keywords:

Graph operations

Graph eigenvalues

Universal adjacency matrix
A B S T R A C T

A new generalization of Fiedler's lemma is obtained by introducing the concept of the main function of a matrix. As applications, the universal spectra of the $H$-join of any graphs (possibly non-regular) and the adjacency spectra of the $H$ generalized join constrained by (arbitrary) vertex subsets are obtained. The adjacency spectra of the generalized corona of graphs is deduced from the spectra of the $H$-join of graphs. Also, the construction of infinitely many pairs of non-regular universal cospectral graphs is provided.

(C) 2021 Elsevier Inc. All rights reserved.

\footnotetext{
The authors would like to thank the anonymous referee for his/her valuable comments and suggestions, which led to an improvement of the original manuscript. The authors would like to thank M. Rajesh Kannan, Department of Mathematics, Indian Institute of Technology, Kharagpur, for his valuable comments and suggestions on this work. The first author would like to thank him for the support and the fruitful discussions during his visit to IIT Kharagpur, which is a motivation for this work. The second author acknowledges the institute postdoctoral fellowship of IISER, Mohali. The third author is grateful to Apoorva Khare, Department of Mathematics, Indian Institute of Science, Bangalore, for his constant support and encouragement. The third author also acknowledges the NBHM grant (0204/7/2019/R\&D-II/6831).

* Corresponding author.

E-mail addresses: dr.msaravanan8187@gmail.com (M. Saravanan), spmath000@gmail.com (S.P. Murugan), arun.maths123@gmail.com, garunkumar@iisc.ac.in (G. Arunkumar).
} 


\section{Introduction}

All the graphs considered in this paper are finite and simple. The eigenvalues of a graph $G$ are the eigenvalues of its adjacency matrix $A(G)$. The set of all eigenvalues of $G$ is called the spectrum of $G$, denoted by $\operatorname{spec}(G)$. For more on graphs and their eigenvalues we refer $[9,10]$. Let $H$ be a graph with vertex set $\left\{v_{1}, v_{2}, \ldots, v_{k}\right\}$ and let $\mathcal{F}=\left\{G_{1}, G_{2}, \ldots, G_{k}\right\}$ be a family of graphs. In [4], the $H$-join operation of the graphs $G_{1}, G_{2}, \ldots, G_{k}$, denoted by $\bigvee \mathcal{F}$, is obtained by replacing the vertex $v_{i}$ of $H$ by the graph $G_{i}$ for $1 \leq i \leq k$ and every vertex of $G_{i}$ is made adjacent with every vertex of $G_{j}$, whenever $v_{i}$ is adjacent to $v_{j}$ in $H$. Precisely, $\bigvee_{H} \mathcal{F}$ is the graph with vertex set $V\left(\bigvee_{H} \mathcal{F}\right)=\bigcup_{i=1}^{k} V\left(G_{i}\right)$ and edge set $E\left(\bigvee_{H} \mathcal{F}\right)=\left(\bigcup_{i=1}^{k} E\left(G_{i}\right)\right) \cup\left(\bigcup_{v_{i} v_{j} \in E(H)}\{x y: x \in\right.$ $\left.\left.V\left(G_{i}\right), y \in V\left(G_{j}\right)\right\}\right)$. In addition to $H$ and $\mathcal{F}$, by considering a family of vertex subsets $\mathcal{S}=\left\{S_{1}, S_{2}, \ldots, S_{k}\right\}$ where $S_{i} \subset V\left(G_{i}\right)$ for each $1 \leq i \leq k$, a generalization of $H$-join operation, known as $H$-generalized join operation constrained by vertex subsets, $\bigvee \mathcal{F}$ is introduced in [5] as follows: $V\left(\bigvee_{H, \mathcal{S}} \mathcal{F}\right)=\bigcup_{i=1}^{k} V\left(G_{i}\right)$ and $E\left(\bigvee_{H, \mathcal{S}} \mathcal{F}\right)=\left(\bigcup_{i=1}^{k} E\left(G_{i}\right)\right) \cup$ ( $\bigcup_{v_{i}, \in E(H)}\left\{x y: x \in S_{i}, y \in S_{j}\right\}$ ). For instance consider the examples in Section 8. If $v_{i} v_{j} \in E(H)$

we take $S_{i}=V\left(G_{i}\right)$ for each $1 \leq i \leq k$, then the $H$-generalized join operation $\bigvee_{H, \mathcal{S}} \mathcal{F}$ coincides with the $H$-join operation of the graphs $G_{1}, G_{2} \ldots, G_{k}$. In [21], the $H$-join operation of the graphs was initially introduced as generalized composition by Schwenk, denoted by $H\left[G_{1}, G_{2}, \ldots, G_{k}\right]$. Also, the same operation is studied in some other names as generalized lexicographic product and joined union in $[19,22,23]$. When all $G_{i}$ 's are equal to the same graph $G$, it is called the lexicographic product [15], denoted by $H[G]$.

The following lemma [12, Lemma 2.2] is proved by M. Fiedler and effectively used in the study of finding sufficient conditions for $k$ arbitrary real numbers to be eigenvalues of a non-negative $k \times k$ symmetric matrix.

Lemma 1. [12] Let $A$ be a symmetric $m \times m$ matrix with eigenvalues $\alpha_{1}, \alpha_{2}, \ldots, \alpha_{m}$ and $B$ be a symmetric $n \times n$ matrix with eigenvalues $\beta_{1}, \beta_{2}, \ldots, \beta_{n}$. Let $u$ be an eigenvector of $A$ corresponding to $\alpha_{1}$ and $v$ be an eigenvector of $B$ corresponding to $\beta_{1}$ such that $\|u\|=\|v\|=1$. Then for any constant $\rho$ the matrix

$$
C=\left[\begin{array}{cc}
A & \rho u v^{t} \\
\rho v u^{t} & B
\end{array}\right]
$$

has eigenvalues $\alpha_{2}, \ldots, \alpha_{m}, \beta_{2}, \ldots, \beta_{n}, \gamma_{1}, \gamma_{2}$ where $\gamma_{1}$ and $\gamma_{2}$ are the eigenvalues of the matrix 


$$
\widehat{C}=\left[\begin{array}{cc}
\alpha_{1} & \rho \\
\rho & \beta_{1}
\end{array}\right]
$$

In $[3,4]$, the above lemma is called Fiedler's lemma. It is easy to see that, this lemma can be used to find the spectrum of $H$-join of regular graphs when $H=K_{2}$. In [3, Lemma 2], a generalization of the Fiedler's Lemma is obtained by Cardoso et al. so that, it can be applied in the $H$-join of regular graphs when $H=P_{k}$, the path graph on $k$ vertices. Then in [4, Theorem 3] Cardoso et al. obtained another improved generalization of Fiedler's lemma as follows, which can be applied in the $H$-join of regular graphs for any $H$.

Theorem 1. [4] Let $M_{i}$ be a symmetric matrix of order $n_{i}$ and $u_{i}$ be an eigenvector of $M_{i}$ corresponding to the eigenvalue $\alpha_{i}$, such that $\left\|u_{i}\right\|=1$ for $1 \leq i \leq k$. Let $\rho_{i, j}$ be a collection of arbitrary scalars such that $\rho_{i, j}=\rho_{j, i}$ for $1 \leq i<j \leq k$. Considering

$$
\mathbf{M}=\left(M_{1}, M_{2}, \ldots, M_{k}\right), \mathbf{u}=\left(u_{1}, u_{2}, \ldots, u_{k}\right)
$$

as $k$-tuples, and

$$
\rho=\left(\rho_{12}, \ldots, \rho_{1, k}, \rho_{23}, \ldots, \rho_{2, k}, \ldots \rho_{k-1 k}\right)
$$

as $\frac{k(k-1)}{2}$-tuple, the following matrices are defined.

$$
\begin{aligned}
& A(\mathbf{M}, \mathbf{u}, \rho):= {\left[\begin{array}{cccc}
M_{1} & \rho_{1,2} u_{1} u_{2}^{t} & \cdots & \rho_{1, k} u_{1} u_{k}^{t} \\
\rho_{2,1} u_{2} u_{1}^{t} & M_{2} & \cdots & \rho_{2, k} u_{2} u_{k}^{t} \\
\vdots & \vdots & \ddots & \vdots \\
\rho_{k, 1} u_{k} u_{1}^{t} & \rho_{k, 2} u_{k} u_{2}^{t} & \cdots & M_{k}
\end{array}\right] \text { and } } \\
& \widehat{A}(\mathbf{M}, \mathbf{u}, \rho):=\left[\begin{array}{cccc}
\alpha_{1} & \rho_{1,2} & \cdots & \rho_{1, k} \\
\rho_{2,1} & \alpha_{2} & \cdots & \rho_{2, k} \\
\vdots & \vdots & \ddots & \vdots \\
\rho_{k, 1} & \rho_{k, 2} & \cdots & \alpha_{k}
\end{array}\right] .
\end{aligned}
$$

Then $\operatorname{spec}(A(\mathbf{M}, \mathbf{u}, \rho))=\left(\bigcup_{i=1}^{k}\left(\operatorname{spec}\left(M_{i}\right) \backslash\left\{\alpha_{i}\right\}\right)\right) \cup \operatorname{spec}(\widehat{A}(\mathbf{M}, \mathbf{u}, \rho))$.

In this paper, we obtain a new generalization of the Fiedler's lemma, in terms of characteristic polynomials as follows. Let $n=\Sigma_{i=1}^{k} n_{i}$. Then

$$
\operatorname{det}\left(\lambda I_{n}-A(\mathbf{M}, \mathbf{u}, \rho)\right)=\left(\Pi_{i=1}^{k} \phi_{i}(\lambda) \Gamma_{i}(\lambda)\right) \operatorname{det}(\widetilde{A}(\mathbf{M}, \mathbf{u}, \rho))
$$

where $\phi_{i}(\lambda)=\operatorname{det}\left(\lambda I_{n_{i}}-M_{i}\right), \Gamma_{i}(\lambda)=u^{t}\left(\lambda I_{n_{i}}-M_{i}\right)^{-1} u$ and 


$$
\widetilde{A}(\mathbf{M}, \mathbf{u}, \rho):=\left[\begin{array}{cccc}
\frac{1}{\Gamma_{1}(\lambda)} & -\rho_{1,2} & \cdots & -\rho_{1, k} \\
-\rho_{2,1} & \frac{1}{\Gamma_{2}(\lambda)} & \cdots & -\rho_{2, k} \\
\vdots & \vdots & \ddots & \vdots \\
-\rho_{k, 1} & -\rho_{k, 2} & \cdots & \frac{1}{\Gamma_{k}(\lambda)}
\end{array}\right] .
$$

The main difference is we are not restricting $\mathbf{u}$ as the $k$-tuple of eigenvectors and $\mathbf{M}$ as the $k$-tuple of symmetric matrices. But, we consider $\mathbf{u}$ as the $k$-tuple of any complex vectors and $\mathbf{M}$ as the $k$-tuple of any complex square matrices of appropriate size. The precious statement is given in Theorem 2. We call the function $\Gamma_{i}(\lambda)$ used above, as the main function of the matrix $M_{i}$. This concept of the main function of a matrix, discussed in Section 2, helps us to accomplish the task. Also as an application of our result, we obtain the characteristic polynomial of $H$-join of graphs when the graphs $G_{i}$ 's are any graphs (possibly non-regular). In [21] it is remarked by Schwenk, that "In general, it does not appear likely that the characteristic polynomial of the generalized composition can always be expressed in terms of the characteristic polynomials of $H, G_{1}, G_{2}, \ldots, G_{k}$ ". In our paper, we prove that it is possible to express the characteristic polynomial of $H$-join operation of graphs (i.e. generalized composition) in terms of the characteristic polynomials and main functions of $G_{1}, G_{2}, \ldots, G_{k}$, and another function obtained from the adjacency matrix of $H$. Moreover for the $H$-join operation of any graphs, we obtain the characteristic polynomial and the spectrum of its universal adjacency matrix.

The universal adjacency matrix of a graph $G$ is defined as follows: Let $A(G), I, J$, and $D(G)$ be the adjacency matrix of $G$, the identity matrix, the all-one matrix, and the degree matrix of $G$, respectively. Any matrix of the form $U(G)=\alpha A+\beta I+\gamma J+\delta D$ where $\alpha, \beta, \gamma, \delta \in \mathbb{R}$ and $\alpha \neq 0$ is called the universal adjacency matrix of $G$. Many interesting and important matrices associated to a graph can be obtained as special cases of $U(G)$. For example, from the universal adjacency matrix $U(G)$, we get adjacency matrix $A(G)$, Laplacian matrix $L(G)=D(G)-A(G)$, signless Laplacian matrix $Q(G)=D(G)+A(G)$, and Seidel matrix $S(G)=J-I-2 A(G)$ by taking appropriate values for $\alpha, \beta, \gamma$, and $\delta$.

In [14], the Laplacian spectra of $H$-join of any graphs is obtained. In [8] the characteristic polynomial of the matrix $A(G)-t D(G)$ is obtained for $H$-join of regular graphs. In [23] the characteristic polynomial of the adjacency matrix of the lexicographic product of any graphs is obtained. Recently in [16] the universal adjacency spectra of the disjoint union of regular graphs is obtained. Also in [5] the eigenvalues of $H$-generalized join operation $\bigvee_{H, \mathcal{S}} \mathcal{F}$ are obtained, when the subsets $S_{i}$ 's are $(\kappa, \tau)$-regular.

In this paper, we obtain the characteristic polynomial and eigenvalues of the universal adjacency matrix of $H$-join of any graphs $G_{1}, G_{2}, \ldots, G_{k}$. Then we obtain the characteristic polynomial and eigenvalues of the adjacency matrix of $H$-generalized join of graphs $G_{1}, G_{2}, \ldots, G_{k}$, where the subsets $S_{i}(G) \subset V\left(G_{i}\right)$ are arbitrary for $1 \leq i \leq k$. Also, we deduce the characteristic polynomial of the generalized corona of graphs by visualizing corona as an $H$-join of graphs. Hence the results obtained (mostly for regular graphs) in $[4,5,8,13,16,23,24]$, are generalized here for any graphs. 
Throughout this paper, we denote the identity matrix of order $n$ by $I_{n}$, the all-one matrix of order $n$ by $J_{n}$ and the all-one vector of size $n \times 1$ by $\mathbf{1}_{n}$.

\section{The main function of a matrix and a generalization of Fiedler's lemma}

Consider a graph $G$ on $n$ vertices with adjacency matrix $A(G)$. Suppose $A(G)$ has spectral decomposition $A(G)=\sum_{i=1}^{k} \theta_{i} E_{\theta_{i}}$, where $\theta_{i}$ 's are distinct eigenvalues of $G$ and $E_{\theta_{i}}$ is the orthogonal projection on the eigenspace of $\theta_{i}, \mathcal{E}\left(\theta_{i}\right)=\operatorname{ker}\left(A(G)-\theta_{i} I_{n}\right)$. An eigenvalue $\theta_{i}$ is called a main eigenvalue if the corresponding eigenspace $\mathcal{E}\left(\theta_{i}\right)$ is not orthogonal to $\mathbf{1}_{n}$. The cosines of the angles between $\mathbf{1}_{n}$ and the eigenspaces of $A$ are known as main angles of $G$, given by $\beta_{i}=\frac{1}{\sqrt{n}}\left\|E_{\theta_{i}} \mathbf{1}_{n}\right\|$, for $1 \leq i \leq k$. So $\theta_{i}$ is a main eigenvalue if and only if $\beta_{i} \neq 0$. For more on the main angles and main eigenvalues, we refer [20] and references therein.

Consider the field of rational functions $\mathbb{C}(\lambda)$. The $\operatorname{det}(\lambda I-A)$ is a non-zero element of $\mathbb{C}(\lambda)$ and hence the matrix $\lambda I-A$ is invertible over $\mathbb{C}(\lambda)$. In [17], the function $\mathbf{1}_{n}^{t}\left(\lambda I_{n}-A(G)\right)^{-1} \mathbf{1}_{n}$ is introduced in the name of coronal of $G$ and is used to find the characteristic polynomial of the corona of two graphs. Since $E_{\theta_{i}}^{2}=E_{\theta_{i}}$, it is easy to see that

$$
\mathbf{1}_{n}^{t}\left(\lambda I_{n}-A(G)\right)^{-1} \mathbf{1}_{n}=\Sigma_{i=1}^{k} \frac{\mathbf{1}_{n}^{t} E_{\theta_{i}} \mathbf{1}_{n}}{\lambda-\theta_{i}}=\Sigma_{i=1}^{k} \frac{\left\|E_{\theta_{i}} \mathbf{1}_{n}\right\|^{2}}{\lambda-\theta_{i}}=\Sigma_{i=1}^{k} \frac{n \beta_{i}^{2}}{\lambda-\theta_{i}},
$$

in which only non-vanishing terms are those terms corresponding to main eigenvalues.

Also in [17], the authors remarked that graphs with different eigenvalues can have the same coronals, meanwhile cospectral graphs can have different coronals. This is because the coronal of a graph depends not only on the eigenvalues but also on the main angles of the graph. In this paper, because of this relationship among main eigenvalues, main angles, and coronal of the graph $G$, we prefer to call $\mathbf{1}_{n}^{t}\left(\lambda I_{n}-A(G)\right)^{-1} \mathbf{1}_{n}$, the main function of the graph $G$, and denote as $\Gamma_{G}(\lambda)$. Moreover for any vectors $u$ and $v$, and a matrix $M$ of the same dimension, we introduce the following notions.

Definition 1. Let $M$ be an $n \times n$ complex matrix, and let $u$ and $v$ be $n \times 1$ complex vectors. The main function associated to the matrix $M$ corresponding to the vectors $u$ and $v$, denoted by $\Gamma_{M}(u, v)$, is defined to be $\Gamma_{M}(u, v ; \lambda)=v^{t}(\lambda I-M)^{-1} u \in \mathbb{C}(\lambda)$. When $u=v$, we denote $\Gamma_{M}(u, v ; \lambda)$ by $\Gamma_{M}(u ; \lambda)$.

Definition 2. Let $M$ be an $n \times n$ normal matrix over $\mathbb{C}$ and let $u$ be an $n \times 1$ complex vector. An eigenvalue $\lambda$ of $M$ is called as $u$-main eigenvalue if the corresponding eigenspace $\mathcal{E}_{M}(\lambda)$ is not orthogonal to the vector $u$. In the case of $u=\mathbf{1}_{n}$, the all-one vector, we don't specify the vector and call eigenvalue $\lambda$ of $M$ as the main eigenvalue of $M$.

Motivated by [15, Theorem 8.13.3], we prove the following lemma. 
Lemma 2. Let $M$ be a complex normal matrix of order $n$ and let $u$ be any $n \times 1$ vector. Then the poles of $u^{t}(\lambda I-M)^{-1} u$ are the u-main eigenvalues of $M$ and are simple.

Proof. Let $\left\{\theta_{1}, \theta_{2}, \cdots, \theta_{k}\right\}$ be the distinct eigenvalues and let $\left\{\theta_{1}, \theta_{2}, \ldots, \theta_{m}\right\}$ be the set of $u$-main eigenvalues of $M$. Suppose the spectral decomposition of $M$ is $M=\Sigma_{i=1}^{k} \theta_{i} E_{\theta_{i}}$, where $E_{\theta_{i}}$ is the orthogonal projection on the eigenspace of $\theta_{i}$. Then $(\lambda I-M)^{-1}=$ $\sum_{i=1}^{k} \frac{E_{\theta_{i}}}{\lambda-\theta_{i}}$, and $\Gamma_{M}(u ; \lambda)=u^{t}(\lambda I-M)^{-1} u=\Sigma_{i=1}^{k} \frac{u^{t} E_{\theta_{i}} u}{\lambda-\theta_{i}}$. Now, $u^{t} E_{\theta_{i}} u \neq 0$ if and only if $\theta_{i}$ is a $u$-main eigenvalue of $M$. So, $\Gamma_{M}(u ; \lambda)=\Sigma_{i=1}^{m} \frac{u^{t} E_{\theta_{i}} u}{\lambda-\theta_{i}}$ and the result follows.

Lemma 3. Let $M$ be a matrix of order $n$ with an eigenvector $u$ corresponding to the eigenvalue $\mu$. Then $\Gamma_{M}(u ; \lambda)=\frac{\|u\|^{2}}{\lambda-\mu}$.

Proof. Now $\left(\lambda I_{n}-M\right) u=(\lambda-\mu) u$. Applying $\left(\lambda I_{n}-M\right)^{-1}$ both sides, we can get $u=\left(\lambda I_{n}-M\right)^{-1}(\lambda-\mu) u$, which implies $\frac{u^{t} u}{\lambda-\mu}=u^{t}\left(\lambda I_{n}-M\right)^{-1} u=\frac{\|u\|^{2}}{\lambda-\mu}$.

Lemma 4. Let $M$ be an $n \times n$ normal matrix over $\mathbb{C}$. Let $u$ be an $n \times 1$ complex vector and $p(M)$ be a polynomial in $M$ with complex coefficients. Then an eigenvalue $\mu$ is a $u$-main eigenvalue of $M$ if and only if $p(\mu)$ is a u-main eigenvalue of $p(M)$.

Proof. For any eigenvalue of $M$ and corresponding eigenvalue of $p(M)$ the eigenvectors are the same. So the eigenspaces are the same and hence the result follows.

Now we can state our main result, a new generalization of Fiedler's lemma.

Theorem 2. Let $M_{i}$ be a complex matrix of order $n_{i}$, and let $u_{i}$ and $v_{i}$ be arbitrary complex vectors of size $n_{i} \times 1$ for $1 \leq i \leq k$. Let $n=\sum_{i=1}^{k} n_{i}$. Let $\rho_{i, j}$ be arbitrary complex numbers for $1 \leq i, j \leq k$ and $i \neq j$. For each $1 \leq i \leq k$, let $\phi_{i}(\lambda)=\operatorname{det}\left(\lambda I_{n_{i}}-M_{i}\right)$ be the characteristic polynomial of the matrix $M_{i}$ and $\Gamma_{i}(\lambda)=\Gamma_{M_{i}}\left(u_{i}, v_{i} ; \lambda\right)=v_{i}^{t}\left(\lambda I-M_{i}\right)^{-1} u_{i}$. Let $\mathbf{M}$ be the $k$-tuple $\left(M_{1}, M_{2}, \ldots, M_{k}\right)$, u be the $2 k$-tuple $\left(u_{1}, v_{1}, u_{2}, v_{2} \ldots, u_{k}, v_{k}\right)$ and $\rho$ be the $k(k-1)$-tuple $\left(\rho_{1,2}, \rho_{1,2} \ldots, \rho_{1, k}, \rho_{2,1}, \rho_{23}, \ldots, \rho_{2, k}, \ldots, \rho_{k, 1}, \rho_{k, 2}, \ldots, \rho_{k-1 k}\right)$. Considering $\mathbf{M}, \mathbf{u}$ and $\rho$, the following matrices are defined:

$$
\begin{gathered}
A(\mathbf{M}, \mathbf{u}, \rho):=\left[\begin{array}{cccc}
M_{1} & \rho_{1,2} u_{1} v_{2}^{t} & \cdots & \rho_{1, k} u_{1} v_{k}^{t} \\
\rho_{2,1} u_{2} v_{1}^{t} & M_{2} & \cdots & \rho_{2, k} u_{2} v_{k}^{t} \\
\vdots & \vdots & \ddots & \vdots \\
\rho_{k, 1} u_{k} v_{1}^{t} & \rho_{k, 2} u_{k} v_{2}^{t} & \cdots & M_{k}
\end{array}\right] \\
\text { and } \widetilde{A}(\mathbf{M}, \mathbf{u}, \rho):=\left[\begin{array}{cccc}
\frac{1}{\Gamma_{1}(\lambda)} & -\rho_{1,2} & \cdots & -\rho_{1, k} \\
-\rho_{2,1} & \frac{1}{\Gamma_{2}(\lambda)} & \cdots & -\rho_{2, k} \\
\vdots & \vdots & \ddots & \vdots \\
-\rho_{k, 1} & -\rho_{k, 2} & \cdots & \frac{1}{\Gamma_{k}(\lambda)}
\end{array}\right] .
\end{gathered}
$$


Then the characteristic polynomial of $A(\mathbf{M}, \mathbf{u}, \rho)$ is given as

$$
\operatorname{det}\left(\lambda I_{n}-A(\mathbf{M}, \mathbf{u}, \rho)\right)=\left(\Pi_{i=1}^{k} \phi_{i}(\lambda) \Gamma_{i}(\lambda)\right) \operatorname{det}(\widetilde{A}(\mathbf{M}, \mathbf{u}, \rho))
$$

Proof of this theorem is given in Section 3.2. At first, we deduce Theorem 1 in terms of characteristic polynomials as a corollary of Theorem 2.

Corollary 1. Consider the notations defined in Theorem 2. Suppose $u_{i}=v_{i}$ is an eigenvector of $M_{i}$ corresponding to an eigenvalue $\alpha_{i}$ with $\left\|u_{i}\right\|=1$, then the characteristic polynomial of $A(\mathbf{M}, \mathbf{u}, \rho)$ is

$$
\begin{gathered}
\phi(A(\mathbf{M}, \mathbf{u}, \rho))=\frac{\phi_{1}}{\lambda-\alpha_{1}} \frac{\phi_{2}}{\lambda-\alpha_{2}} \ldots \frac{\phi_{k}}{\lambda-\alpha_{k}} \operatorname{det}(\widetilde{A}(\mathbf{M}, \mathbf{u}, \rho)) \\
\text { where } \widetilde{A}(\mathbf{M}, \mathbf{u}, \rho)=\left[\begin{array}{cccc}
\lambda-\alpha_{1} & -\rho_{1,2} & \cdots & -\rho_{1, k} \\
-\rho_{2,1} & \lambda-\alpha_{2} & \cdots & -\rho_{2, k} \\
\vdots & \vdots & \ddots & \vdots \\
-\rho_{k, 1} & -\rho_{k, 2} & \cdots & \lambda-\alpha_{k}
\end{array}\right] .
\end{gathered}
$$

Proof. Since $\left\|u_{i}\right\|=1$, by Lemma 3 we get $\Gamma_{i}=\frac{1}{\lambda-\alpha_{i}}$. Now the proof follows from Theorem 2.

In [8, Theorem 2.3] another generalization of Fiedler's lemma, similar to Theorem 1, is given for the matrices with fixed row sum and the result is used to find the generalized characteristic polynomial of $H$-join of regular graphs. We observe that any such matrix has the all-one vector as an eigenvector. By taking $u_{i}$ to be the all-one vector of appropriate size, we can deduce [8, Theorem 2.3] from Theorem 2.

\section{Proof of the main result}

In this section, we prove Theorem 2. We start with the following essential lemmas.

\subsection{Some important lemmas}

Lemma 5. [9] Let $A, B, C$ and $D$ be matrices such that $M=\left[\begin{array}{ll}A & B \\ C & D\end{array}\right]$. If $D$ is invertible, then $\operatorname{det}(M)=\operatorname{det}(D) \operatorname{det}\left(A-B D^{-1} C\right)$.

Lemma 6. [1,11] Let $A$ be an $n \times n$ invertible matrix, and let $u$ and $v$ be any two $n \times 1$ vectors such that $1+v^{t} A^{-1} u \neq 0$. Then

(1) $\operatorname{det}\left(A+u v^{t}\right)=\left(1+v^{t} A^{-1} u\right) \operatorname{det}(A)$. 
(2) $\left(A+u v^{t}\right)^{-1}=A^{-1}-\frac{A^{-1} u v^{t} A^{-1}}{1+v^{t} A^{-1} u}$.

Lemma 7. Let $A$ be an $n \times n$ complex matrix, and let $u$ and $v$ be any $n \times 1$ complex vectors. Also, let $\Gamma=v^{t}(\lambda I-A)^{-1} u$. Then

(1) $\operatorname{det}\left(\lambda I-A+\alpha u v^{t}\right)=(1+\alpha \Gamma) \operatorname{det}(\lambda I-A)=(1+\alpha \Gamma) \phi_{A}(\lambda)$

(2) $v^{t}\left(\lambda I-A+\alpha u v^{t}\right) u=\frac{\Gamma}{1+\alpha \Gamma}$

Proof. The proof of (1) follows directly from Lemma 6(1), as $\operatorname{det}\left(\lambda I-A+\alpha u v^{t}\right)=$ $\left(1+\alpha v^{t}(\lambda I-A)^{-1} u\right) \operatorname{det}(\lambda I-A)$. So we prove (2).

By Lemma 6(2),

$$
\left(\lambda I-A+\alpha u v^{t}\right)^{-1}=(\lambda I-A)^{-1}-\alpha \frac{(\lambda I-A)^{-1} u v^{t}(\lambda I-A)^{-1}}{1+\alpha v^{t}(\lambda I-A)^{-1} u}
$$

which implies,

$$
v^{t}\left(\lambda I-A+\alpha u v^{t}\right)^{-1} u=\Gamma-\alpha \frac{\Gamma^{2}}{1+\alpha \Gamma}=\frac{\Gamma}{1+\alpha \Gamma}
$$

\subsection{Proof of Theorem 2}

Proof. We prove the result by using induction on $k$. For convenience, we take $\Gamma_{i}=$ $\Gamma_{i}(\lambda)$. The base case $k=1$ is clear. We prove the result also for $k=2$ for the sake of understanding. Now, by Lemma 5 , we have

$$
\begin{aligned}
\left|\begin{array}{cc}
\lambda I_{n_{1}}-M_{1} & -\rho_{1,2} u_{1} v_{2}^{t} \\
-\rho_{2,1} u_{2} v_{1}^{t} & \lambda I_{n_{2}}-M_{2}
\end{array}\right| & =\operatorname{det}\left(\lambda I_{n_{2}}-M_{2}\right) \operatorname{det}\left(\lambda I_{n_{1}}-M_{1}-\rho_{1,2} \rho_{2,1} \Gamma_{2} u_{1} v_{1}^{t}\right) \\
& =\phi_{1} \phi_{2}\left(1-\rho_{1,2} \rho_{2,1} \Gamma_{2} \Gamma_{1}\right), \text { by Lemma } 7(1) \\
& =\phi_{1} \phi_{2}\left|\begin{array}{cc}
1 & -\rho_{1,2} \Gamma_{1} \\
-\rho_{2,1} \Gamma_{2} & 1
\end{array}\right|
\end{aligned}
$$

This proves the result for the case $k=2$. We assume the result is true for $k-1$. Consider the matrix

$$
\lambda I_{n}-A(\mathbf{M}, \mathbf{u}, \rho)=\left[\begin{array}{cccc}
\lambda I_{n_{1}}-M_{1} & -\rho_{1,2} u_{1} v_{2}^{t} & \cdots & -\rho_{1, k} u_{1} v_{k}^{t} \\
-\rho_{2,1} u_{2} v_{1}^{t} & \lambda I_{n_{2}}-M_{2} & \cdots & -\rho_{2, k} u_{2} v_{k}^{t} \\
\vdots & \vdots & \ddots & \vdots \\
-\rho_{k, 1} u_{k} v_{1}^{t} & -\rho_{k, 2} u_{k} v_{2}^{t} & \cdots & \lambda I_{n_{k}}-M_{k}
\end{array}\right]
$$

Now, by Lemma 5, we have

$$
\operatorname{det}\left(\lambda I_{n}-A(\mathbf{M}, \mathbf{u}, \rho)\right)=\operatorname{det}\left(\lambda I_{n_{k}}-M_{k}\right) \operatorname{det}(S)
$$


where

$$
\begin{aligned}
S= & {\left[\begin{array}{cccc}
\lambda I_{n_{1}}-M_{1} & -\rho_{1,2} u_{1} v_{2}^{t} & \cdots & -\rho_{1, k-1} u_{1} v_{k-1}^{t} \\
-\rho_{2,1} u_{2} v_{1}^{t} & \lambda I_{n_{2}}-M_{2} & \cdots & -\rho_{2, k-1} u_{2} v_{k-1}^{t} \\
\vdots & \vdots & \ddots & \vdots \\
-\rho_{k-1,1} u_{k-1} v_{1}^{t} & -\rho_{k-1,2} u_{k-1} v_{2}^{t} & \cdots & \lambda I_{n_{k-1}}-M_{k-1}
\end{array}\right] } \\
& -\left[\begin{array}{c}
-\rho_{1, k} u_{1} v_{k}^{t} \\
-\rho_{2, k} u_{2} v_{k}^{t} \\
\vdots \\
-\rho_{k-1, k} u_{k-1} v_{k}^{t}
\end{array}\right]\left(\lambda I_{n_{k}}-M_{k}\right)^{-1}\left[\begin{array}{llll}
-\rho_{k, 1} u_{k} v_{1}^{t} & -\rho_{k, 2} u_{k} v_{2}^{t} & \cdots & -\rho_{k, k-1} u_{k} v_{k-1}^{t}
\end{array}\right] \\
= & {\left[s_{i j}\right] }
\end{aligned}
$$

given by $s_{i j}=\left\{\begin{array}{l}\lambda I_{n_{i}}-M_{i}-\Gamma_{k} \rho_{i, k} \rho_{k, i} u_{i} v_{i}^{t} \text { if } i=j \\ -\left(\rho_{i, j}+\Gamma_{k} \rho_{i, k} \rho_{k, j}\right) u_{i} v_{j}^{t} \text { if } i \neq j\end{array}\right.$.

By Lemma $7, \operatorname{det}\left(\lambda I_{n_{i}}-M_{i}-\Gamma_{k} \rho_{i, k} \rho_{k, i} u_{i} v_{i}^{t}\right)=\operatorname{det}\left(\lambda I_{n_{i}}-M_{i}\right)\left(1-\left(\Gamma_{k} \rho_{i, k} \rho_{k, i}\right) \Gamma_{i}\right)$ and $v_{i}^{t}\left(\lambda I_{n_{i}}-M_{i}-\Gamma_{k} \rho_{i, k} \rho_{k, i} u_{i} v_{i}^{t}\right)^{-1} u_{i}=\frac{\Gamma_{i}}{1-\left(\Gamma_{k} \rho_{i, k} \rho_{k, i}\right) \Gamma_{i}}$.

Now by the induction hypothesis on $S$ we get

$$
\begin{aligned}
\operatorname{det}(S) & =\left(\Pi_{i=1}^{k-1} \operatorname{det}\left(\lambda I_{n_{i}}-M_{i}-\Gamma_{k} \rho_{i, k} \rho_{k, i} u_{i} v_{i}^{t}\right) \frac{\Gamma_{i}}{1-\left(\Gamma_{k} \rho_{i, k} \rho_{k, i}\right) \Gamma_{i}}\right) \operatorname{det}(\widetilde{S}) \\
& =\left(\Pi_{i=1}^{k-1} \operatorname{det}\left(\lambda I_{n_{i}}-M_{i}\right) \Gamma_{i}\right) \operatorname{det}(\widetilde{S})
\end{aligned}
$$

where $\widetilde{S}=\left[\widetilde{s}_{i j}\right]$ given by $\widetilde{s}_{i j}=\left\{\begin{array}{l}\frac{1-\rho_{i, k} \rho_{k, i} \Gamma_{k} \Gamma_{i}}{\Gamma_{i}} \text { if } i=j \\ -\left(\rho_{i, j}+\Gamma_{k} \rho_{i, k} \rho_{k, j}\right) \text { if } i \neq j\end{array}\right.$

Therefore

$$
\begin{aligned}
& \operatorname{det}(S)=\phi_{1} \phi_{2} \cdots \phi_{k-1} \\
& \times\left|\begin{array}{cccc}
1-\left(\rho_{1, k} \rho_{k, 1} \Gamma_{k} \Gamma_{1}\right) & -\left(\rho_{1,2}+\Gamma_{k} \rho_{1, k} \rho_{k, 2}\right) \Gamma_{1} & \cdots & -\left(\rho_{1 k-1}+\Gamma_{k} \rho_{1, k} \rho_{k, k-1}\right) \Gamma_{1} \\
-\left(\rho_{2,1}+\Gamma_{k} \rho_{2, k} \rho_{k, 1}\right) \Gamma_{2} & 1-\left(\rho_{2, k} \rho_{k, 2} \Gamma_{k} \Gamma_{2}\right) & \cdots & -\left(\rho_{2 k-1}+\Gamma_{k} \rho_{2, k} \rho_{k, k-1}\right) \Gamma_{2} \\
\vdots & \vdots & \ddots & \vdots \\
-\left(\rho_{k-1,1}+\Gamma_{k} \rho_{k-1, k} \rho_{k, 1}\right) \Gamma_{k-1} & -\left(\rho_{k-1,2}+\Gamma_{k} \rho_{k-1, k} \rho_{k, 2}\right) \Gamma_{k-1} & \cdots & 1-\left(\rho_{k-1, k} \rho_{k, k-1} \Gamma_{k} \Gamma_{k-1}\right)
\end{array}\right| \\
& =\phi_{1} \phi_{2} \cdots \phi_{k-1} \times\left(\left|\begin{array}{cccc}
1 & -\rho_{1,2} \Gamma_{1} & \cdots & -\rho_{1 k-1} \Gamma_{1} \\
-\rho_{2,1} \Gamma_{2} & 1 & \cdots & -\rho_{2 k-1} \Gamma_{2} \\
\vdots & \vdots & \vdots & \\
-\rho_{k-1,1} \Gamma_{k-1} & -\rho_{k-1,2} \Gamma_{k-1} & \cdots & 1
\end{array}\right|\right. \\
& \left.-\left|\begin{array}{cccc}
-\rho_{1, k} \rho_{k, 1} \Gamma_{k} \Gamma_{1} & -\rho_{1, k} \rho_{k, 2} \Gamma_{k} \Gamma_{1} & \cdots & -\rho_{1, k} \rho_{k, k-1} \Gamma_{k} \Gamma_{1} \\
-\rho_{2, k} \rho_{k, 1} \Gamma_{k} \Gamma_{2} & -\rho_{2, k} \rho_{k, 2} \Gamma_{k} \Gamma_{2} & \cdots & -\rho_{2, k} \rho_{k, k-1} \Gamma_{k} \Gamma_{2} \\
\vdots & \vdots & \vdots & \\
-\rho_{k-1, k} \rho_{k, 1} \Gamma_{k} \Gamma_{k-1} & -\rho_{k-1, k} \rho_{k, 2} \Gamma_{k} \Gamma_{k-1} & \cdots & -\rho_{k-1, k} \rho_{k, k-1} \Gamma_{k} \Gamma_{k-1}
\end{array}\right|\right)
\end{aligned}
$$




$$
=\phi_{1} \phi_{2} \cdots \phi_{k-1} \times\left|\begin{array}{ccccc}
1 & -\rho_{1,2} \Gamma_{1} & \cdots & -\rho_{1, k-1} \Gamma_{1} & -\rho_{1, k} \Gamma_{1} \\
-\rho_{2,1} \Gamma_{2} & 1 & \cdots & -\rho_{2, k-1} \Gamma_{2} & -\rho_{2, k} \Gamma_{2} \\
\vdots & \vdots & \vdots & \vdots & \vdots \\
-\rho_{k-1,1} \Gamma_{k-1} & -\rho_{k-1,2} \Gamma_{k-1} & \cdots & 1 & -\rho_{k-1, k} \Gamma_{k-1} \\
-\rho_{k, 1} \Gamma_{k} & -\rho_{k, 2} \Gamma_{k} & \cdots & -\rho_{k, k-1} \Gamma_{k} & 1
\end{array}\right|,
$$

again by Lemma 5 .

By substituting this $\operatorname{det}(S)$ value in Equation (3.1), we get the required result for $k$. This completes the proof of Theorem 2.

Suppose the matrices $M_{i}$ 's are normal and $\left\{\theta_{1}, \theta_{2}, \ldots, \theta_{m_{i}}\right\}$ is the set of distinct $u_{i^{-}}$ main eigenvalues of $M_{i}$, for $1 \leq i \leq k$. Then as discussed in the proof of Lemma 2, we can write

$$
\Gamma_{i}=\frac{f_{i}}{g_{i}} \text { where } g_{i}=\prod_{j=1}^{m_{i}}\left(\lambda-\theta_{j}\right)
$$

Hence by the Theorem 2,

$$
\operatorname{det}(\lambda I-A(\mathbf{M}, \mathbf{u}, \rho))=\left(\frac{\phi_{1}}{g_{1}}\right) \ldots\left(\frac{\phi_{k}}{g_{k}}\right) \Phi(\lambda)
$$

where $\Phi(\lambda)=\left|\begin{array}{cccc}g_{1}(\lambda) & -\rho_{1,2} f_{1}(\lambda) & \cdots & -\rho_{1, k} f_{1}(\lambda) \\ -\rho_{2,1} f_{2}(\lambda) & g_{2}(\lambda) & \cdots & -\rho_{2, k} f_{2}(\lambda) \\ \vdots & \vdots & \ddots & \vdots \\ -\rho_{k, 1} f_{k}(\lambda) & -\rho_{k, 2} f_{k}(\lambda) & \cdots & g_{k}(\lambda)\end{array}\right|$. So we can describe the spectrum of $A(\mathbf{M}, \mathbf{u}, \rho)$ as follows.

Theorem 3. Consider the notations defined above. Suppose the matrices $M_{i}$ 's are normal, then

- Every eigenvalue, which is not a $u_{i}$-main eigenvalue of $M_{i}$, say $\lambda$ with multiplicity $m(\lambda)$ is an eigenvalue of $A(\mathbf{M}, \mathbf{u}, \rho)$ with multiplicity $m(\lambda)$.

- Every $u_{i}$-main eigenvalue of $M_{i}$, say $\lambda$ with multiplicity $m(\lambda)$ is an eigenvalue of $A(\mathbf{M}, \mathbf{u}, \rho)$ with multiplicity $m(\lambda)-1$.

- Remaining eigenvalues are the roots of the polynomial $\Phi(\lambda)$.

Proof. By Lemma 2 the poles of $\Gamma_{i}$ are $u_{i^{-}}$main eigenvalues and they are simple. Now the proof easily follows from Equation (3.3).

\section{Universal spectra of the $\boldsymbol{H}$-join of graphs}

In this section, by applying Theorem 2, we obtain the results on characteristic polynomial and spectrum of the universal adjacency matrix of $H$-join of graphs. 
Consider a graph $H$ on $k$ vertices and a family of graphs $\mathcal{F}=\left\{G_{1}, G_{2}, \ldots, G_{k}\right\}$. Let $G=\bigvee_{H} \mathcal{F}$ be the $H$-join of graphs in $\mathcal{F}$, and let $n_{i}, A_{i}$ and $D_{i}$ be the number of vertices, the adjacency matrix and the degree matrix of the graph $G_{i}$ respectively, for $1 \leq i \leq k$. Also let $\rho_{i, j}$ be the scalars defined by $\rho_{i, j}=\rho_{j, i}=1$ if $i j \in E(H)$ and 0 otherwise, for $1 \leq i, j \leq k$ and $i \neq j$. Once and for all we fix the partition of $V(G)$ as $V\left(G_{1}\right) \cup V\left(G_{2}\right) \cup \cdots \cup V\left(G_{k}\right)$, so that the adjacency matrix of the graph $G$ is given by

$$
A(G)=\left[\begin{array}{cccc}
A_{1} & \rho_{1,2} \mathbf{1}_{n_{1}} \mathbf{1}_{n_{2}}^{t} & \cdots & \rho_{1, k} \mathbf{1}_{n_{1}} \mathbf{1}_{n_{k}}^{t} \\
\rho_{2,1} \mathbf{1}_{n_{2}} \mathbf{1}_{n_{1}}^{t} & A_{2} & \cdots & \rho_{2, k} \mathbf{1}_{n_{2}} \mathbf{1}_{n_{k}}^{t} \\
\vdots & \vdots & \ddots & \vdots \\
\rho_{k, 1} \mathbf{1}_{n_{k}} \mathbf{1}_{n_{1}}^{t} & \rho_{k, 2} \mathbf{1}_{n_{k}} \mathbf{1}_{n_{2}}^{t} & \cdots & A_{k}
\end{array}\right]
$$

\subsection{Universal spectra of the H-join of graphs}

The proof of the following Observation is immediate from the definition of the $H$-join of graphs.

Observation 1. Let $H$ be a graph with vertex set $\left\{v_{1}, \ldots, v_{k}\right\}$ and $\mathcal{F}=\left\{G_{1}, G_{2}, \ldots, G_{k}\right\}$ be a family of $k$ graphs such that $V\left(G_{i}\right)=\left\{v_{1}^{(i)}, \ldots, v_{n_{i}}^{(i)}\right\}$ for $1 \leq i \leq k$. Then the degree of the vertex $v_{j}^{(i)}$ in $G=\bigvee_{H} \mathcal{F}$ is given by

$$
\operatorname{deg}_{G}\left(v_{j}^{(i)}\right)=\operatorname{deg}_{G_{i}}\left(v_{j}^{(i)}\right)+w_{i}, 1 \leq i \leq k, 1 \leq j \leq n_{i}
$$

where $w_{i}=\sum_{v_{l} \in N_{H}\left(v_{i}\right)} n_{l}$.

Let $U(G)=\alpha A(G)+\beta I_{n}+\gamma J_{n}+\delta D(G)$ with $\alpha \neq 0$ be the universal adjacency matrix of the graph $G$, where $n=\Sigma_{i=1}^{k} n_{i}$. Let $U_{i}=\alpha A_{i}+\beta I_{n_{i}}+\gamma J_{n_{i}}+\delta D_{i}$ be the universal adjacency matrix of the graph $G_{i}$, for $1 \leq i \leq k$. Therefore by the Equation (4.1) the universal adjacency matrix of $G$ can be written as follows:

$$
U(G)=\left[\begin{array}{cccc}
U_{1}+\delta w_{1} I_{n_{1}} & \left(\rho_{1,2} \alpha+\gamma\right) \mathbf{1}_{n_{1}} \mathbf{1}_{n_{2}}^{t} & \cdots & \left(\rho_{1, k} \alpha+\gamma\right) \mathbf{1}_{n_{1}} \mathbf{1}_{n_{k}}^{t} \\
\left(\rho_{2,1} \alpha+\gamma\right) \mathbf{1}_{n_{2}} \mathbf{1}_{n_{1}}^{t} & U_{2}+\delta w_{2} I_{n_{2}} & \cdots & \left(\rho_{2, k} \alpha+\gamma\right) \mathbf{1}_{n_{2}} \mathbf{1}_{n_{k}}^{t} \\
\vdots & \vdots & \ddots & \vdots \\
\left(\rho_{k, 1} \alpha+\gamma\right) \mathbf{1}_{n_{k}} \mathbf{1}_{n_{1}}^{t} & \left(\rho_{k, 2} \alpha+\gamma\right) \mathbf{1}_{n_{k}} \mathbf{1}_{n_{2}}^{t} & \cdots & U_{k}+\delta w_{k} I_{n_{k}}
\end{array}\right]
$$

In the following theorem, we obtain the characteristic polynomial of universal adjacency matrix $U(G)$.

Theorem 4. Let $H$ be a graph on $k$ vertices and $\mathcal{F}=\left\{G_{1}, G_{2}, \ldots, G_{k}\right\}$ be a family of any graphs. Consider the graph $G=\bigvee_{H} \mathcal{F}$. Let $\phi_{i}(\lambda)$ be the characteristic polynomial of $U_{i}$ and $\Gamma_{i}(\lambda)=\Gamma_{U_{i}}\left(\mathbf{1}_{n_{i}} ; \lambda\right)$. Then we have the following. 
i) The characteristic polynomial of the universal adjacency matrix $U(G)$ given in Equation (4.2) is

$$
\begin{aligned}
& \phi_{U(G)}(\lambda)=\left(\Pi_{i=1}^{k} \phi_{i}\left(\lambda-\delta w_{i}\right) \Gamma_{i}\left(\lambda-\delta w_{i}\right)\right) \operatorname{det}(\widetilde{U}(G)) \\
& \text { where } \widetilde{U}(G)=\left[\begin{array}{cccc}
\frac{1}{\Gamma_{1}\left(\lambda-\delta w_{1}\right)} & -\left(\rho_{1,2} \alpha+\gamma\right) & \cdots & -\left(\rho_{1, k} \alpha+\gamma\right) \\
-\left(\rho_{2,1} \alpha+\gamma\right) & \frac{1}{\Gamma_{2}\left(\lambda-\delta w_{2}\right)} & \cdots & -\left(\rho_{2, k} \alpha+\gamma\right) \\
\vdots & \vdots & \ddots & \vdots \\
-\left(\rho_{k, 1} \alpha+\gamma\right) & -\left(\rho_{k, 2} \alpha+\gamma\right) & \cdots & \frac{1}{\Gamma_{k}\left(\lambda-\delta w_{k}\right)}
\end{array}\right]
\end{aligned}
$$

ii) Analogous to the Equations (3.2) and (3.3), we define $f_{i}, g_{i}$, and $\Phi(\lambda)$ corresponding to the main eigenvalues of $U_{i}$ for $1 \leq i \leq k$. Then the universal spectrum of $G$ is given as below.

- For every eigenvalue $\mu$ of $U_{i}$ with multiplicity $m(\mu)$, which is not a main eigenvalue, $\mu+\delta w_{i}$ is a universal eigenvalue of $G$ with multiplicity $m(\mu)$.

- For every main eigenvalue $\mu$ of $U_{i}$ with multiplicity $m(\mu), \mu+\delta w_{i}$ is a universal eigenvalue of $G$ with multiplicity $m(\mu)-1$.

- Remaining eigenvalues are the roots of the polynomial $\Phi(\lambda)$.

Proof. For each $1 \leq i \leq k$, let $P_{i}=U_{i}+\delta w_{i} I_{n_{i}}$. Then we have the following relations,

$$
\begin{gathered}
\phi_{P_{i}}(\lambda)=\operatorname{det}\left(\lambda I_{n_{i}}-\left(U_{i}+\delta w_{i} I_{n_{i}}\right)\right)=\phi_{U_{i}}\left(\lambda-\delta w_{i}\right) \text { and } \\
\Gamma_{P_{i}}(\lambda)=\mathbf{1}_{n_{i}}^{t}\left(\lambda I_{n_{i}}-\left(U_{i}+\delta w_{i} I_{n_{i}}\right)\right)^{-1} \mathbf{1}_{n_{i}}=\Gamma_{U_{i}}\left(\lambda-\delta w_{i}\right) .
\end{gathered}
$$

Let $\widehat{\rho}_{i, j}=\widehat{\rho}_{j, i}=\rho_{i, j} \alpha+\gamma$ for $1 \leq i<j \leq k$. Considering the triplet $(\mathbf{M}, \mathbf{u}, \widehat{\rho})$, given by

$$
\begin{gathered}
\mathbf{M}=\left(P_{1}, P_{2}, \ldots, P_{k}\right), \mathbf{u}=\left(\mathbf{1}_{n_{1}}, \mathbf{1}_{n_{2}} \ldots, \mathbf{1}_{n_{k}}\right) \text { and } \\
\widehat{\rho}=\left(\widehat{\rho}_{1,2}, \ldots, \widehat{\rho}_{1, k}, \widehat{\rho}_{23}, \ldots, \widehat{\rho}_{2, k}, \ldots, \widehat{\rho}_{k-1, k}\right)
\end{gathered}
$$

we can write the matrices in the Equations (4.2) and (4.3) as $U(G)=A(\mathbf{M}, \mathbf{u}, \widehat{\rho})$ and $\widetilde{U}(G)=\widetilde{A}(\mathbf{M}, \mathbf{u}, \widehat{\rho})$. Now using Theorem 2 the proof of (i) follows. By Lemma $4, \mu$ is not a main eigenvalue of $U_{i}$ if and only if $\mu+\delta w_{i}$ is not a main eigenvalue of $P_{i}$. Now by Theorem 3 the proof of (ii) follows.

Corollary 2. Consider the notations defined in Theorem 4. Suppose the graph $G_{i}$ is $r_{i^{-}}$ regular for $1 \leq i \leq k$. Then $p_{i}=\alpha r_{i}+\beta+\gamma n_{i}+\delta\left(r_{i}+w_{i}\right)$ is an eigenvalue of $P_{i}=U_{i}+\delta w_{i} I_{n_{i}}$ and

$$
\operatorname{spec}(U(G))=\left(\bigcup_{i=1}^{k}\left(\operatorname{spec}\left(P_{i}\right) \backslash\left\{p_{i}\right\}\right)\right) \cup \operatorname{spec}\left(\widetilde{U^{\prime}}(G)\right)
$$


where $\widetilde{U^{\prime}}(G)=\left[\begin{array}{cccc}p_{1} & \sqrt{n_{1} n_{2}} \widehat{\rho}_{1,2} & \cdots & \sqrt{n_{1} n_{k}} \widehat{\rho}_{1, k} \\ \sqrt{n_{2} n_{1}} \widehat{\rho}_{2,1} & p_{2} & \cdots & \sqrt{n_{2} n_{k}} \widehat{\rho}_{2, k} \\ \vdots & \vdots & \ddots & \vdots \\ \sqrt{n_{k} n_{1}} \widehat{\rho}_{k, 1} & \sqrt{n_{k} n_{2}} \widehat{\rho}_{k, 2} & \cdots & p_{k}\end{array}\right]$.

Proof. Clearly $\mathbf{1}_{n_{i}}$ is an eigenvector of $P_{i}$ corresponding to the eigenvalue $p_{i}=\alpha r_{i}+$ $\beta+\gamma n_{i}+\delta\left(r_{i}+w_{i}\right)$. Let $\phi_{i}=\operatorname{det}\left(\lambda I_{n_{i}}-P_{i}\right)$ and $\Gamma_{i}=\Gamma_{P_{i}}\left(\mathbf{1}_{n_{i}} ; \lambda\right)$. Now, by Lemma 3 , we have $\Gamma_{i}=\frac{n_{i}}{\lambda-p_{i}}$ and so by Theorem 4, we get

$$
\phi(U(G))=\frac{\phi_{1}}{\lambda-p_{1}} \frac{\phi_{2}}{\lambda-p_{2}} \cdots \frac{\phi_{k}}{\lambda-p_{k}} n_{1} n_{2} \cdots n_{k} \operatorname{det}(\widetilde{U}(G)) .
$$

Distributing $n_{i}$ inside the determinant of $\widetilde{U}(G)$, as $\sqrt{n_{i}}$ into the $i^{\text {th }}$ row and $\sqrt{n_{i}}$ into the $i^{\text {th }}$ column, we can write

$$
\begin{aligned}
n_{1} n_{2} \cdots n_{k} \operatorname{det}(\widetilde{U}(G)) & =\operatorname{det}\left[\begin{array}{cccc}
\left(\lambda-p_{1}\right) & -\sqrt{n_{1} n_{2}} \widehat{\rho}_{1,2} & \cdots & -\sqrt{n_{1} n_{k}} \widehat{\rho}_{1, k} \\
-\sqrt{n_{2} n_{1}} \widehat{\rho}_{2,1} & \left(\lambda-p_{2}\right) & \cdots & -\sqrt{n_{2} n_{k}} \widehat{\rho}_{2, k} \\
\vdots & \vdots & \ddots & \vdots \\
-\sqrt{n_{k} n_{1}} \widehat{\rho}_{k, 1} & -\sqrt{n_{k} n_{2}} \widehat{\rho}_{k, 2} & \cdots & \left(\lambda-p_{k}\right)
\end{array}\right] \\
& =\operatorname{det}\left(\lambda I_{n}-\widetilde{U^{\prime}}(G)\right) .
\end{aligned}
$$

Now the proof follows.

Corollary 3. Consider the notations defined in Theorem 4. Assume that $\alpha+\delta=0$. Then $p_{i}=\beta+\gamma n_{i}+\delta w_{i}$ is an eigenvalue of $P_{i}=U_{i}+\delta w_{i} I_{n_{i}}$ and

$$
\operatorname{spec}(U(G))=\left(\bigcup_{i=1}^{k}\left(\operatorname{spec}\left(P_{i}\right) \backslash\left\{p_{i}\right\}\right)\right) \cup \operatorname{spec}\left(\widetilde{U^{\prime}}(G)\right)
$$

where $\widetilde{U^{\prime}}(G)=\left[\begin{array}{cccc}p_{1} & \sqrt{n_{1} n_{2}} \widehat{\rho}_{1,2} & \cdots & \sqrt{n_{1} n_{k}} \widehat{\rho}_{1, k} \\ \sqrt{n_{2} n_{1}} \widehat{\rho}_{2,1} & p_{2} & \cdots & \sqrt{n_{2} n_{k}} \widehat{\rho}_{2, k} \\ \vdots & \vdots & \ddots & \vdots \\ \sqrt{n_{k} n_{1}} \widehat{\rho}_{k, 1} & \sqrt{n_{k} n_{2}} \widehat{\rho}_{k, 2} & \cdots & p_{k}\end{array}\right]$.

Proof. It is easy to see that

$$
\left(\alpha A_{i}+\beta I_{n_{i}}+\gamma J_{n_{i}}+\delta D_{i}\right) \mathbf{1}_{n_{i}}=(\alpha+\delta)\left[\begin{array}{c}
\operatorname{deg}_{G_{i}}\left(v_{1}^{(i)}\right) \\
\operatorname{deg}_{G_{i}}\left(v_{2}^{(i)}\right) \\
\vdots \\
\operatorname{deg}_{G_{i}}\left(v_{n_{i}}^{(i)}\right)
\end{array}\right]+\left(\beta+\gamma n_{i}\right) \mathbf{1}_{n_{i}} .
$$

So $\mathbf{1}_{n_{i}}$ is an eigenvector of $P_{i}$ corresponding to the eigenvalue $p_{i}=\beta+\gamma n_{i}+\delta w_{i}$. Then by the same argument as in the previous corollary, the proof follows. 
Remark 1. In [4, Theorem 8], the authors obtained the Laplacian spectra of $H$-join of any graphs. For a given graph $G$, the Laplacian matrix $L(G)$, is obtained from the universal adjacency matrix $U(G)$, by taking $(\alpha, \beta, \gamma, \delta)=(-1,0,0,1)$. So, we can deduce [4, Theorem 8] from Corollary 3.

Let $H$ be a graph on $k$ vertices and $G^{\prime}$ be any graph. We recall that the Lexicographic product of graphs $H$ and $G^{\prime}$, denoted by $H\left[G^{\prime}\right]$, is obtained as the $H$-join of graphs in $\mathcal{F}=\left\{G_{1}, G_{2}, \ldots, G_{k}\right\}$, where $G_{i}=G^{\prime}$ for $1 \leq i \leq k$. In [23], the authors obtained the characteristic polynomial of $H\left[G^{\prime}\right]$ and investigated the spectrum in various cases. Now we generalize [23, Theorem 2.4] by obtaining the characteristic polynomial of the universal adjacency matrix of $H\left[G^{\prime}\right]$ when $\delta=0$.

Theorem 5. Let $H$ be a graph on $k$ vertices and $G^{\prime}$ be a graph on $n^{\prime}$ vertices. Consider the graph $G=H\left[G^{\prime}\right]$, the lexicographic product of $H$ and $G^{\prime}$. Suppose $\operatorname{spec}(H)=$ $\left\{\lambda_{1}, \lambda_{2}, \ldots, \lambda_{k}\right\}$. Then the characteristic polynomial of the universal adjacency matrix of $U(G)$ when $\delta=0$, is

$$
\phi_{U(G)}(\lambda)=\phi^{k}(\lambda)\left(\Pi_{i=1}^{k}\left(1-\lambda_{i} \Gamma(\lambda)\right)\right)
$$

where $\phi(\lambda)$ is the characteristic polynomial of $U\left(G^{\prime}\right)$ and $\Gamma(\lambda)=\Gamma_{U\left(G^{\prime}\right)}\left(\mathbf{1}_{n^{\prime}} ; \lambda\right)$ when $\delta=0$.

Proof. By Theorem 4,

$$
\begin{aligned}
\phi_{U(G)}(\lambda) & =\phi^{k}(\lambda) \Gamma^{k}(\lambda) \operatorname{det}\left(\frac{1}{\Gamma(\lambda)} I_{k}-A(H)\right) \\
& =\phi^{k}(\lambda) \Gamma^{k}(\lambda)\left(\Pi_{i=1}^{k}\left(\frac{1}{\Gamma(\lambda)}-\lambda_{i}\right)\right) \\
& =\phi^{k}(\lambda)\left(\Pi_{i=1}^{k}\left(1-\lambda_{i} \Gamma(\lambda)\right)\right) \cdot
\end{aligned}
$$

\subsection{The generalized characteristic polynomial of the $H$-join of graphs}

The generalized characteristic polynomial of a graph $G$ is introduced in [9], as the bivariate polynomial defined by $\phi_{G}(\lambda, t)=\operatorname{det}(\lambda I-(A(G)-t D(G)))$ where $A(G)$ and $D(G)$ are the adjacency and the degree matrix associated to the graph $G$. As mentioned earlier, in [8, Theorem 3.1] the authors obtained a generalization of Fiedler's lemma, for the matrices with fixed row sum and as an application, they obtained the generalized characteristic polynomial of $H$-join of regular graphs. In the following theorem, we obtain the generalized characteristic polynomial of $H$-join of any graphs. 
Theorem 6. Let $H$ be any graph and $\mathcal{F}=\left\{G_{1}, G_{2}, \ldots, G_{k}\right\}$ be a family of any $k$ graphs. Consider the graph $G=\bigvee_{H} \mathcal{F}$. Let $M(G)=A(G)-t D(G)$ and $M_{i}=A_{i}-t D_{i}$ for $1 \leq i \leq k$. Let $\phi_{i}$ be the characteristic polynomial of $M_{i}$ and $\Gamma_{i}=\Gamma_{M_{i}}\left(\mathbf{1}_{n_{i}} ; \lambda\right)$. Then

i) The generalized characteristic polynomial of the graph $G$ is

$$
\phi_{M(G)}(\lambda)=\left(\Pi_{i=1}^{k} \phi_{i}\left(\lambda+t w_{i}\right) \Gamma_{i}\left(\lambda+t w_{i}\right)\right) \operatorname{det}(\widetilde{M}(G))
$$

where $\widetilde{M}(G)=\left[\begin{array}{cccc}\frac{1}{\Gamma_{1}\left(\lambda+t w_{1}\right)} & -\rho_{1,2} & \cdots & -\rho_{1, k} \\ -\rho_{2,1} & \frac{1}{\Gamma_{2}\left(\lambda+t w_{2}\right)} & \cdots & -\rho_{2, k} \\ \vdots & \vdots & \ddots & \vdots \\ -\rho_{k, 1} & -\rho_{k, 2} & \cdots & \frac{1}{\Gamma_{k}\left(\lambda+t w_{3}\right)}\end{array}\right]$.

ii) Analogous to the Equations (3.2) and (3.3), we define $f_{i}, g_{i}$, and $\Phi(\lambda)$ corresponding to the main eigenvalues of $M_{i}$ for $1 \leq i \leq k$. Then the spectrum of $M(G)$ is given below.

- For every eigenvalue $\mu$ of $M_{i}$ with multiplicity $m(\mu)$, which is not a main eigenvalue, $\mu-t_{i}$ is an eigenvalue of $M(G)$ with multiplicity $m(\mu)$.

- For every main eigenvalue $\mu$ of $M_{i}$ with multiplicity $m(\mu), \mu-t w_{i}$ is an eigenvalue of $M(G)$ with multiplicity $m(\mu)-1$.

- Remaining eigenvalues are the roots of the polynomial $\Phi(\lambda)$.

Proof. The proof directly follows from Theorem 4, by taking $(\alpha, \beta, \gamma, \delta)=(1,0,0,-t)$ in the universal adjacency matrix $U(G)$.

Remark 2. Suppose the graph $G_{i}$ is $r_{i}$-regular for each $1 \leq i \leq k$. Then $p_{i}=r_{i}-t\left(r_{i}+w_{i}\right)$ is an eigenvalue of $P_{i}=M_{i}-t w_{i} I_{n_{i}}$. Similar to the proof of the Corollary 2 of Theorem 4, here also we obtain the following result on spectrum of $M(G)$.

$$
\operatorname{spec}(M(G))=\left(\bigcup_{i=1}^{k}\left(\operatorname{spec}\left(P_{i}\right) \backslash\left\{p_{i}\right\}\right)\right) \cup \operatorname{spec}\left(\widetilde{M^{\prime}}(G)\right)
$$

where $\widetilde{M^{\prime}}(G)=\left[\begin{array}{cccc}p_{1} & \sqrt{n_{1} n_{2}} \rho_{1,2} & \cdots & \sqrt{n_{1} n_{k}} \rho_{1, k} \\ \sqrt{n_{2} n_{1}} \rho_{2,1} & p_{2} & \cdots & \sqrt{n_{2} n_{k}} \rho_{2, k} \\ \vdots & \vdots & \ddots & \vdots \\ \sqrt{n_{k} n_{1}} \rho_{k, 1} & \sqrt{n_{k} n_{2}} \rho_{k, 2} & \cdots & p_{k}\end{array}\right]$.

This result is one of the main results of [8].

Remark 3. In [8, Corollary 3.2], from the generalized characteristic polynomial, the characteristic polynomial of the adjacency matrix, the Laplacian matrix, the signless Laplacian matrix, and the normalized Laplacian matrix are deduced for $H$-join of regular graphs. Similarly, we can also deduce them for $H$-join of any graphs from Theorem 6 . 
Also, we can deduce the characteristic polynomial of the Seidel adjacency matrix of $H$ join of any graphs by taking $(\alpha, \beta, \gamma, \delta)=(-2,-1,1,0)$ in the universal adjacency matrix $U(G)$ in Theorem 4 .

Lemma 8. [17] Let $G$ be a complete bipartite graph $K_{p, q}$ on $p+q=n$ vertices. Then $\Gamma_{A(G)}\left(\mathbf{1}_{n} ; \lambda\right)=\frac{n \lambda+2 p q}{\lambda^{2}-p q}$.

Corollary 4. Let $H$ be a graph on $k$ vertices and $\mathcal{F}=\left\{G_{1}, G_{2}, \ldots, G_{k}\right\}$ be a family of $k$ graphs such that $G_{i}=K_{p_{i}, q_{i}}$ for $1 \leq i \leq k$. Consider the graph $G=\bigvee_{H} \mathcal{F}$. Let $n=\Sigma_{i=1}^{k}\left(p_{i}+q_{i}\right)$ be the number of vertices of $G$. Then the spectrum of $G$ is given as below.

- 0 is an eigenvalue with multiplicity $n-2 k$.

- The remaining eigenvalues are the roots of the polynomial $\Phi(\lambda)$, where

$$
\Phi(\lambda)=\operatorname{det}\left[\begin{array}{cccc}
\lambda^{2}-p_{1} q_{1} & -\rho_{1,2}\left(n_{1} \lambda+2 p_{1} q_{1}\right) & \cdots & -\rho_{1, k}\left(n_{1} \lambda+2 p_{1} q_{1}\right) \\
-\rho_{2,1}\left(n_{2} \lambda+2 p_{2} q_{2}\right) & \lambda^{2}-p_{2} q_{2} & \cdots & -\rho_{2, k}\left(n_{2} \lambda+2 p_{2} q_{2}\right) \\
\vdots & \vdots & \ddots & \vdots \\
-\rho_{k, 1}\left(n_{k} \lambda+2 p_{k} q_{k}\right) & -\rho_{k, 2}\left(n_{k} \lambda+2 p_{k} q_{k}\right) & \cdots & \lambda^{2}-p_{k} q_{k}
\end{array}\right]
$$

Proof. Let $\phi_{i}(\lambda)=\operatorname{det}\left(\lambda I_{n_{i}}-A\left(G_{i}\right)\right)$ and $\Gamma_{i}(\lambda)=\Gamma_{A\left(G_{i}\right)}\left(\mathbf{1}_{n_{i}} ; \lambda\right)$ where $n_{i}=p_{i}+q_{i}$. By Lemma $8, \Gamma_{i}(\lambda)=\frac{n_{i} \lambda+2 p_{i} q_{i}}{\lambda^{2}-p_{i} q_{i}}$. By taking $t=0$ in Theorem 6 , the characteristic polynomial of $A(G)$ is given as

$$
\phi_{A(G)}(\lambda)=\left(\Pi_{i=1}^{k} \frac{\phi_{i}(\lambda)}{\lambda^{2}-p_{i} q_{i}}\right) \operatorname{det}(\widetilde{A}(G))
$$

where $\widetilde{A}(G)=\left[\begin{array}{cccc}\lambda^{2}-p_{1} q_{1} & -\rho_{1,2}\left(n_{1} \lambda+2 p_{1} q_{1}\right) & \cdots & -\rho_{1, k}\left(n_{1} \lambda+2 p_{1} q_{1}\right) \\ -\rho_{2,1}\left(n_{2} \lambda+2 p_{2} q_{2}\right) & \lambda^{2}-p_{2} q_{2} & \cdots & -\rho_{2, k}\left(n_{2} \lambda+2 p_{2} q_{2}\right) \\ \vdots & \vdots & \ddots & \vdots \\ -\rho_{k, 1}\left(n_{k} \lambda+2 p_{k} q_{k}\right) & -\rho_{k, 2}\left(n_{k} \lambda+2 p_{k} q_{k}\right) & \cdots & \lambda^{2}-p_{k} q_{k}\end{array}\right]$.

Then the proof follows from the fact that $\phi_{i}(\lambda)=\lambda^{n_{i}-2}\left(\lambda^{2}-p_{i} q_{i}\right)$ for $1 \leq i \leq k$.

Remark 4. The above result can be generalized by replacing the family $\mathcal{F}$ of complete bipartite graphs with any family of graphs having exactly two main eigenvalues.

\section{Cospectral graphs}

Two graphs are said to be cospectral if their (adjacency) spectrum are equal. In general, for any matrix say $M(G)$ for a given graph $G$, two graphs are said to be $M$ cospectral if their $M$-spectrum is equal. In [2], the author questioned the existence of 
non-regular graphs, which are cospectral with respect to the adjacency, the Laplacian, the signless Laplacian, and the normalized Laplacian spectrum simultaneously. In [8, Theorem 3.7], the authors affirmatively answered the question by the construction of such graphs using the $H$-join of regular graphs. In the following theorem, we prove that those graphs are $U$-cospectral too. In particular, those graphs are cospectral with respect to the Seidel spectrum also.

Lemma 9. If $G_{1}$ and $G_{2}$ are cospectral $r$-regular graphs on $n$ vertices, then $\operatorname{spec}\left(A\left(G_{1}\right)+\right.$ $\left.J_{n}\right)=\operatorname{spec}\left(A\left(G_{2}\right)+J_{n}\right)$.

Proof. $\mathbf{1}_{n}$ is an eigenvector of $A\left(G_{1}\right)+J_{n}$ and $A\left(G_{2}\right)+J_{n}$ corresponding to the eigenvalue $r+n$. Every eigenvector different from $\mathbf{1}_{n}$ of $G_{1}$ and $G_{2}$ lies in the kernel of $J_{n}$. Hence the result follows.

Theorem 7. Let $\mathcal{F}=\mathcal{F}_{1}=\left\{G_{1}, G_{2}, \ldots, G_{k}\right\}$ and $\mathcal{F}_{2}=\left\{G_{1}^{\prime}, G_{2}^{\prime}, \ldots, G_{k}^{\prime}\right\}$ be two families of graphs.

(i) If $G_{i}$ and $G_{i}^{\prime}$ are cospectral regular graphs on $n_{i}$ vertices for $1 \leq i \leq k$, and $H$ is an arbitrary graph on $k$ vertices, then $\bigvee_{H} \mathcal{F}_{1}$ and $\bigvee_{H} \mathcal{F}_{2}$ are $U$-cospectral.

(ii) If $H_{1}$ and $H_{2}$ are cospectral $r_{1}$-regular graphs on $k$ vertices and every $G_{i}$ is $r_{2}$ regular on $m$ vertices for $1 \leq i \leq k$, then $\bigvee_{H_{1}} \mathcal{F}$ and $\bigvee_{H_{2}} \mathcal{F}$ are $U$-cospectral.

(iii) If $H_{1}$ and $H_{2}$ are cospectral $r_{1}$-regular graphs on $k$ vertices and, $G_{i}$ and $G_{i}^{\prime}$ are cospectral $r_{2}$-regular graphs on $m$ vertices for $1 \leq i \leq k$ then $\bigvee_{H_{1}} \mathcal{F}_{1}$ and $\bigvee_{H_{2}} \mathcal{F}_{2}$ are $U$-cospectral.

Proof. Let $G=\bigvee_{H} \mathcal{F}_{1}$ and $G^{\prime}=\bigvee_{H} \mathcal{F}_{2}$. Let $U_{i}$ and $U_{i}^{\prime}$ be the universal adjacency matrix of $G_{i}$ and $G_{i}^{\prime}$ respectively. Let $P_{i}=U_{i}+\delta w_{i} I_{n_{i}}$ and $P_{i}^{\prime}=U_{i}^{\prime}+\delta w_{i} I_{n_{i}}$ where $w_{i}$ is defined as in Observation 1 . Then by Lemma $9, \operatorname{spec}\left(P_{i}\right)=\operatorname{spec}\left(P_{i}^{\prime}\right)$ for $1 \leq i \leq k$. Now by Corollary 2, $\operatorname{spec}(U(G))=\operatorname{spec}\left(U\left(G^{\prime}\right)\right)$, since $\widetilde{U^{\prime}}(G)=\widetilde{U^{\prime}}\left(G^{\prime}\right)$. Thus (i) is proved.

Let $G=\bigvee_{H_{1}} \mathcal{F}$ and $G^{\prime}=\bigvee_{H_{2}} \mathcal{F}$. Let $U_{i}$ be the universal adjacency matrix of $G_{i}$. Since $H_{1}$ and $H_{2}$ are $r_{1}$-regular, $w_{i}=r_{1} m$ and $p_{i}=\alpha r_{2}+\beta+\gamma m+\delta\left(r_{2}+r_{1} m\right)$ for $1 \leq i \leq k$. By Lemma 9, $\operatorname{spec}\left(\alpha A\left(H_{1}\right)+\gamma\left(J_{k}-I_{k}\right)\right)=\operatorname{spec}\left(\alpha A\left(H_{2}\right)+\gamma\left(J_{k}-I_{k}\right)\right)$. Now by Corollary 2 , $\operatorname{spec}(U(G))=\operatorname{spec}\left(U\left(G^{\prime}\right)\right)$, since $\widetilde{U^{\prime}}(G)$ and $\widetilde{U^{\prime}}\left(G^{\prime}\right)$ are cospectral. Thus (ii) is proved.

To prove (iii), let $G=\bigvee_{H_{1}} \mathcal{F}_{1}$ and $G^{\prime}=\bigvee_{H_{2}} \mathcal{F}_{2}$. By the same arguments as in (i) and (ii), we get $P_{i}$ and $P_{i}^{\prime}$ are cospectral and, $\widetilde{U^{\prime}}(G)$ and $\widetilde{U^{\prime}}\left(G^{\prime}\right)$ are cospectral. Thus the proof of (iii) follows. 


\section{Spectra of the $H$-generalized join of graphs}

In this section, we obtain the characteristic polynomial of $H$-generalized join of graphs $\bigvee \mathcal{F}$ introduced in [5].

$H, \mathcal{S}$

Definition 3. Let $G$ be any graph. A vertex subset $S$ of a graph $G$ is said to be $(\kappa, \tau)$ regular if $S$ induces a $\kappa$-regular graph in $G$ and every vertex outside of $S$ has $\tau$ neighbours in $S$. When $G$ is a regular graph, for convenience $S=V(G)$ is considered as $(\kappa, 0)$-regular.

Definition 4. Let $G$ be any graph with vertex set $\left\{v_{1}, v_{2}, \ldots, v_{n}\right\}$. For any subset $S \subset$ $V(G)$, the characteristic vector of $S$, denoted by $\chi_{S}$, is defined as the $0-1$ vector such that $i^{\text {th }}$ place of $\chi_{S}$ is 1 if and only if the vertex $v_{i} \in S$.

Lemma 10. [6,18] Let $G$ be an $r$-regular graph on $n$ vertices with a $(\kappa, \tau)$-regular set $S$. Then $\kappa-\tau \in \operatorname{spec}(G)$ and $\mathbf{v}=(r-\kappa) \chi_{S}-\tau \chi_{\bar{S}}$ is an eigenvector corresponding to $\kappa-\tau$, where $\bar{S}=V(G) \backslash S$.

Lemma 11. [7] Let $G$ be a graph with a $(\kappa, \tau)$-regular set $S$, where $\tau>0$, and $\lambda \in$ $\sigma(A(G))$. Then, $\lambda$ is not a main eigenvalue of $G$ if and only if $\lambda=\kappa-\tau$ or $\chi_{S} \in$ $\left(\mathcal{E}_{G}(\lambda)\right)^{\perp}$.

Fix a $(\kappa, \tau)$-regular subset $S$ of $V(G)$. An eigenvalue $\lambda \in \sigma(G)$ is said to be a special eigenvalue if $\lambda \neq \kappa-\tau$ and $\lambda$ is not a main eigenvalue. Then by Lemma 11, if $\lambda$ is a special eigenvalue of $G$ then $\lambda$ is not a $\chi_{s}$-main eigenvalue. In [5] the authors obtained all eigenvalues of $\bigvee_{H, \mathcal{S}} \mathcal{F}$ when $G_{i}$ is regular and the subsets $S_{i} \in \mathcal{S}$ are such that $S_{i}=V\left(G_{i}\right)$ for $1 \leq i \leq k$, in which case $\bigvee_{H, \mathcal{S}} \mathcal{F}$ coincides with the $H$-join of regular graphs $\bigvee_{H} \mathcal{F}$. In other cases, it is proved that every special eigenvalue corresponding to $\left(\kappa_{i}, \tau_{i}\right)$-regular subset $S_{i}$ is an eigenvalue of $\bigvee_{H, \mathcal{S}} \mathcal{F}$ and thus the partial spectrum is obtained for $\bigvee_{H, \mathcal{S}} \mathcal{F}$.

In the following theorem, we obtain the characteristic polynomial of $\bigvee_{H, \mathcal{S}} \mathcal{F}$ for any family of subsets $\mathcal{S}$ and obtain the complete set of eigenvalues.

Theorem 8. Consider a graph $H$ of order $k$ and a family of graphs $\mathcal{F}=\left\{G_{1}, \ldots, G_{k}\right\}$. Consider also a family of vertex subsets $\mathcal{S}=\left\{S_{1}, \ldots, S_{k}\right\}$, such that $S_{i} \subset V\left(G_{i}\right)$ for $1 \leq i \leq k$. Let $G=\bigvee_{H, S} \mathcal{F}$. Let $n_{i}$ and $A_{i}$ be the number of vertices and the adjacency matrix of the graph $G_{i}$ respectively for $1 \leq i \leq k$. For $1 \leq i, j \leq k$, let $\rho_{i, j}$ be the scalars defined by $\rho_{i, j}=1$ if $i j \in E(H)$ and 0 otherwise. Then we have the following. 
i) The characteristic polynomial of $G$ is

$$
\begin{gathered}
\phi_{G}(\lambda)=\left(\prod_{i=1}^{k} \phi_{i}(\lambda) \Gamma_{i}(\lambda)\right) \operatorname{det}(\widetilde{A}(G)) \\
\text { where } \widetilde{A}(G)=\left[\begin{array}{cccc}
\frac{1}{\Gamma_{1}} & -\rho_{1,2} & \cdots & -\rho_{1, k} \\
-\rho_{2,1} & \frac{1}{\Gamma_{2}} & \cdots & -\rho_{2, k} \\
\vdots & \vdots & \ddots & \vdots \\
-\rho_{k, 1} & -\rho_{k, 2} & \cdots & \frac{1}{\Gamma_{k}}
\end{array}\right]
\end{gathered}
$$

where $\phi_{i}(\lambda)=\operatorname{det}\left(\lambda I_{n_{i}}-A\left(G_{i}\right)\right)$ and $\Gamma_{i}(\lambda)=\Gamma_{A_{i}}\left(\chi_{S_{i}} ; \lambda\right)$.

ii) Analogous to the Equations (3.2) and (3.3), we define $f_{i}, g_{i}$ and $\Phi(\lambda)$ corresponding to the $\chi_{S_{i}}$-main eigenvalues of $G_{i}$ for $1 \leq i \leq k$. Then the spectrum of $G$ is given as below.

- Every eigenvalue $\mu$ of $A_{i}$ with multiplicity $m(\mu)$, which is not $\chi_{S_{i}}$-main eigenvalue, is an eigenvalue of $G$ with multiplicity $m(\mu)$.

- Every $\chi_{S_{i}}$-main eigenvalue $\mu$ of $A_{i}$ with multiplicity $m(\mu)$, is an eigenvalue of $G$ with multiplicity $m(\mu)-1$.

- Remaining eigenvalues are the roots of the polynomial $\Phi(\lambda)$.

Proof. By the definition of $\bigvee_{(H, S)} \mathcal{F}$, the adjacency matrix of $G$ is given as

$$
A(G)=\left[\begin{array}{cccc}
A_{1} & \rho_{1,2} \chi_{S_{1}} \chi_{S_{2}}^{t} & \cdots & \rho_{1, k} \chi_{S_{1}} \chi_{S_{k}}^{t} \\
\rho_{2,1} \chi_{S_{2}} \chi_{S_{1}}^{t} & A_{2} & \cdots & \rho_{2, k} \chi_{S_{2}} \chi_{S_{k}}^{t} \\
\vdots & \vdots & \ddots & \vdots \\
\rho_{k, 1} \chi_{S_{k}} \chi_{S_{1}}^{t} & \rho_{k, 2} \chi_{S_{k}} \chi_{S_{2}}^{t} & \cdots & A_{k}
\end{array}\right]
$$

Then by direct application of Theorem 2 and Theorem 3 on $A(G)$, the proofs of (i) and (ii) follow immediately.

Lemma 12. Let $G$ be an $r$-regular graph on $n$ vertices. Let $S$ be a $(\kappa, \tau)$-regular set of $G$. Suppose $\kappa-\tau$ is a simple eigenvalue of $G$. Then $\Gamma_{A(G)}\left(\chi_{S} ; \lambda\right)=\frac{|S|^{2}}{n(\lambda-r)}+$ $\frac{|S|^{2}(r-\kappa)^{2}}{c(\lambda-(\kappa-\tau))}$, where $c=(r-\kappa)^{2}|S|+\tau^{2}(n-|S|)$.

Proof. By Lemma 10, $\kappa-\tau$ is an eigenvalue of $G$ with corresponding eigenvector $\mathbf{v}=(r-$ $\kappa) \chi_{S}-\tau \chi_{\bar{S}}$. Every eigenvector corresponding to an eigenvalue $\lambda$ such that $\kappa-\tau \neq \lambda \neq r$, is orthogonal to the vector $\chi_{S}$. Thus the only $\chi_{S}$-main eigenvalues of $G$ are $\kappa-\tau$ and $r$. Let the orthogonal projection on the eigenspace corresponding to the eigenvalue $r$ be $E_{r}$ and to the eigenvalue $\kappa-\tau$ be $E_{\kappa-\tau}$. Then $E_{r}=\frac{1}{n} \mathbf{1}_{n} \mathbf{1}_{n}^{t}$ and $E_{\kappa-\tau}=\frac{1}{c} \mathbf{v} \mathbf{v}^{t}$, 
where $c=(r-\kappa)^{2}|S|+\tau^{2}|\bar{S}|$. Therefore, $\Gamma_{A(G)}\left(\chi_{S} ; \lambda\right)=\frac{\chi_{S}^{t} E_{r} \chi_{S}}{\lambda-r}+\frac{\chi_{S}^{t} E_{\kappa-\tau} \chi_{S}}{\lambda-(\kappa-\tau)}=$ $\frac{|S|^{2}}{n(\lambda-r)}+\frac{|S|^{2}(r-\kappa)^{2}}{c(\lambda-(\kappa-\tau))}$.

Corollary 5. Consider a graph $H$ of order $k$ and a family of graphs $\mathcal{F}=\left\{G_{1}, \ldots, G_{k}\right\}$, where $G_{i}$ is $r_{i}$-regular graph on $n_{i}$ vertices for $1 \leq i \leq k$. Consider also a family of vertex subsets $\mathcal{S}=\left\{S_{1}, \ldots, S_{k}\right\}$, such that $S_{i} \in V\left(G_{i}\right)$ is a $\left(\kappa_{i}, \tau_{i}\right)$-regular set and, $\kappa_{i}-\tau_{i}$ is a simple eigenvalue of $G_{i}$ for $1 \leq i \leq k$. Then

$$
\Gamma_{i}(\lambda)=\Gamma_{A\left(G_{i}\right)}\left(\chi_{S_{i}} ; \lambda\right)=\frac{\left|S_{i}\right|^{2}}{n\left(\lambda-r_{i}\right)}+\frac{\left|S_{i}\right|^{2}\left(r_{i}-\kappa_{i}\right)^{2}}{c_{i}\left(\lambda-\left(\kappa_{i}-\tau_{i}\right)\right)},
$$

where $c_{i}=\left(r_{i}-\kappa_{i}\right)^{2}\left|S_{i}\right|+\tau_{i}^{2}\left(n_{i}-\left|S_{i}\right|\right)$. Let $g_{i}=\left(\lambda-r_{i}\right)\left(\lambda-\left(\kappa_{i}-\tau_{i}\right)\right)$ and $f_{i}=\Gamma_{i}(\lambda) g_{i}$. Let $G=\bigvee_{H, S} \mathcal{F}$. The spectrum of $G$ is given as

$$
\operatorname{spec}(G)=\left(\bigcup_{i=1}^{k}\left(\operatorname{spec}\left(G_{i}\right) \backslash\left\{r_{i}, k_{i}-\tau_{i}\right\}\right)\right) \cup\left(\bigcup_{i=1}^{k}\left\{x_{1}^{(i)}, x_{2}^{(i)}\right\}\right)
$$

where $x_{1}^{(1)}, x_{2}^{(1)}, x_{1}^{(2)}, x_{2}^{(2)}, \ldots, x_{1}^{(k)}$ and $x_{2}^{(k)}$ are the roots of the polynomial

$$
\Phi(\lambda)=\operatorname{det}\left[\begin{array}{cccc}
g_{1} & -\rho_{1,2} f_{1} & \cdots & -\rho_{1, k} f_{1} \\
-\rho_{2,1} f_{2} & g_{2} & \cdots & -\rho_{2, k} f_{2} \\
\vdots & \vdots & \ddots & \vdots \\
-\rho_{k, 1} f_{k} & -\rho_{k, 2} f_{k} & \cdots & g_{k}
\end{array}\right] .
$$

Proof. The expression of $\Gamma_{i}(\lambda)$ follows from Lemma 12. The remaining proof is obtained from Theorem 8, by the similar arguments used in the proof of Corollary 4.

\section{Spectra of the generalized corona of graphs}

In [13, Theorem 3.1], the generalized corona product is defined as below and its characteristic polynomial is obtained. In this section, we deduce this result as a corollary of Theorem 2. This is done by viewing the corona product as the $H$-join of suitably chosen graphs.

Definition 5. Let $H^{\prime}$ be a graph on $k$ vertices. Let $G_{1}, G_{2}, \ldots, G_{k}$ be graphs of order $n_{1}, n_{2}, \ldots, n_{k}$ respectively. The generalized corona product of $H^{\prime}$ with $G_{1}, G_{2}, \ldots, G_{k}$, denoted by $H^{\prime} \tilde{o} \Lambda_{i=1}^{k} G_{i}$, is obtained by taking one copy of graphs $H^{\prime}, G_{1}, G_{2}, \ldots, G_{k}$, and joining the $i^{t h}$ vertex of $H^{\prime}$ to every vertex of $G_{i}$.

When $G_{i}=G^{\prime}$ for all $i$, the graph $H^{\prime} \tilde{o} \Lambda_{i=1}^{k} G_{i}$ is called simply corona of $H^{\prime}$ and $G^{\prime}$, denoted by $H^{\prime} \circ G^{\prime}$. 
Theorem 9. Let $H^{\prime}$ be a graph with vertex set $V\left(H^{\prime}\right)=\left\{v_{1}, v_{2}, \ldots, v_{k}\right\}$. Let $G_{1}, G_{2}, \ldots$, $G_{k}$ be any graphs. $\rho_{i, j}=1$ if $v_{i} v_{j} \in E\left(H^{\prime}\right)$ and 0 otherwise. The characteristic polynomial of the generalized corona product $G=H^{\prime} \tilde{o} \Lambda_{i=1}^{k} G_{i}$ is given by

$$
\phi_{G}(\lambda)=\left(\Pi_{i=1}^{k} \phi_{G_{i}}(\lambda)\right) \operatorname{det}\left(\widetilde{A}\left(H^{\prime}\right)\right)
$$

where $\widetilde{A}\left(H^{\prime}\right)=\left[\begin{array}{cccc}\lambda-\Gamma_{G_{1}}(\lambda) & -\rho_{1,2} & \cdots & -\rho_{1, k} \\ -\rho_{2,1} & \lambda-\Gamma_{G_{2}}(\lambda) & \cdots & -\rho_{2, k} \\ \vdots & \vdots & \ddots & \vdots \\ -\rho_{k, 1} & -\rho_{k, 2} & \cdots & \lambda-\Gamma_{G_{k}}(\lambda)\end{array}\right]$.

Proof. Let $H=H^{\prime} \circ K_{1}$. Let $v_{k+i}$ be the new vertex in $H$ attached with the vertex $v_{i}$ in the copy of $H^{\prime}$, for $1 \leq i \leq k$. Let $\mathcal{F}=\left\{K_{1}, K_{1}, \ldots, K_{1}, G_{1}, G_{2}, \ldots, G_{k}\right\}$. Then we get the following visualization of generalized corona as $H$-join of graphs in $\mathcal{F}$.

$$
\left(H^{\prime} \tilde{\circ} \Lambda_{i=1}^{k} G_{i}\right)=\left(\bigvee_{H} \mathcal{F}\right)
$$

That is, each $v_{i}$ is replaced by $K_{1}$ and $v_{k+i}$ is replaced by $G_{i}$ in $H$, to form the $H$-join.

Now $A(H)=\left[\begin{array}{cc}A\left(H^{\prime}\right) & I_{k} \\ I_{k} & 0_{k}\end{array}\right]$. Since $\phi_{K_{1}}(\lambda)=\lambda$ and $\Gamma_{K_{1}}(\lambda)=\frac{1}{\lambda}$, by letting $\alpha=1$, $\beta=\gamma=\delta=0$ in Theorem 4, we get

$$
\phi_{G}(\lambda)=\left(\Pi_{i=1}^{k}\left(\phi_{K_{1}}(\lambda) \phi_{G_{i}}(\lambda) \Gamma_{K_{1}}(\lambda) \Gamma_{G_{i}}(\lambda)\right)\right) \operatorname{det}(\widetilde{A}(H))
$$

which implies

$$
\phi_{G}(\lambda)=\left(\Pi_{i=1}^{k}\left(\phi_{G_{i}}(\lambda) \Gamma_{G_{i}}(\lambda)\right)\right) \operatorname{det}(\widetilde{A}(H))
$$

where $\widetilde{A}(H)=\left[\begin{array}{c}\lambda I_{k}-A\left(H^{\prime}\right) \\ -I_{k}\end{array} \operatorname{diag}\left(\frac{1}{\Gamma_{G_{1}}(\lambda)}, \frac{-I_{k}}{\Gamma_{G_{2}}(\lambda)}, \ldots, \frac{1}{\Gamma_{G_{k}}(\lambda)}\right)\right]$. Now by Lemma 5 , $\operatorname{det}(\widetilde{A}(H))$ is given as

$\operatorname{det}\left[\begin{array}{cccc}\frac{1}{\Gamma_{G_{1}}(\lambda)} & 0 & \cdots & 0 \\ 0 & \frac{1}{\Gamma_{G_{2}}(\lambda)} & \cdots & 0 \\ \vdots & \vdots & \ddots & \vdots \\ 0 & 0 & \cdots & \frac{1}{\Gamma_{G_{k}}(\lambda)}\end{array}\right] \operatorname{det}\left(\lambda I_{k}-A\left(H^{\prime}\right)-\left[\begin{array}{cccc}\Gamma_{G_{1}}(\lambda) & 0 & \cdots & 0 \\ 0 & \Gamma_{G_{2}}(\lambda) & \cdots & 0 \\ \vdots & \vdots & \ddots & \vdots \\ 0 & 0 & \cdots & \left.\Gamma_{G_{k}}(\lambda)\right)\end{array}\right]\right)$ 
$=\left(\Pi_{i=1}^{k} \frac{1}{\Gamma_{G_{i}}(\lambda)}\right) \operatorname{det}\left(\left[\begin{array}{cccc}\lambda-\Gamma_{G_{1}}(\lambda) & 0 & \cdots & 0 \\ 0 & \lambda-\Gamma_{G_{2}}(\lambda) & \cdots & 0 \\ \vdots & \vdots & \ddots & \vdots \\ 0 & 0 & \cdots & \left.\lambda-\Gamma_{G_{k}}(\lambda)\right)\end{array}\right]-A\left(H^{\prime}\right)\right)$

Now by substituting $\operatorname{det}(\widetilde{A}(H))$ in Equation (7.1) we get the required result.

Remark 5. Similarly, we can get the other variants of the spectra of the generalized corona of any graphs. The same work can be done on other variants of corona also by suitable choice of $H$.

\section{Examples}

To illustrate our results, we compute the characteristic polynomials of two particular examples on $H$-join of graphs and $H$-generalized join of graphs constrained by vertex subsets. Similarly, we can apply our other results also.

Example 1. Consider the graphs $H=P_{3}, G_{1}=P_{3}, G_{2}=K_{1,3}$ and $G_{3}=K_{2}$ as follows.

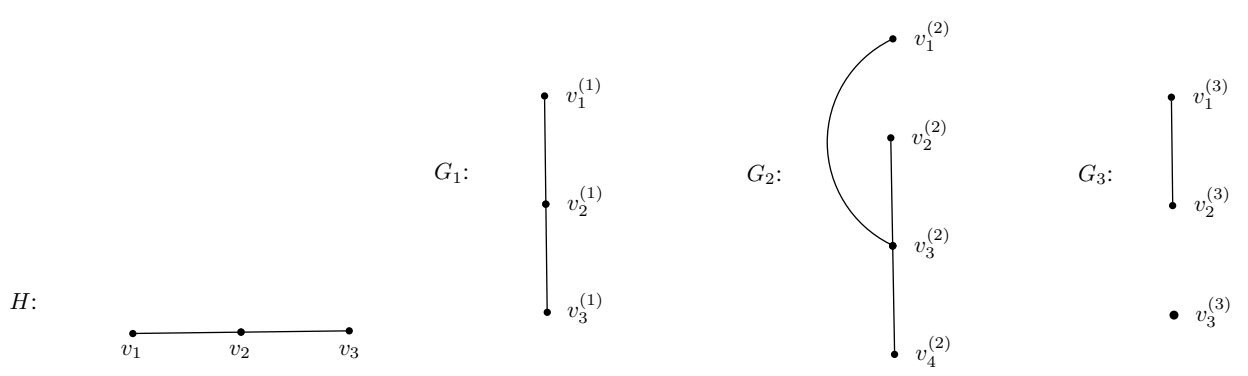

Let $\mathcal{F}=\left\{G_{1}, G_{2}, G_{3}\right\}$. Then the $H$-join graph $G=\bigvee_{H} \mathcal{F}$ is given as

$G$ :

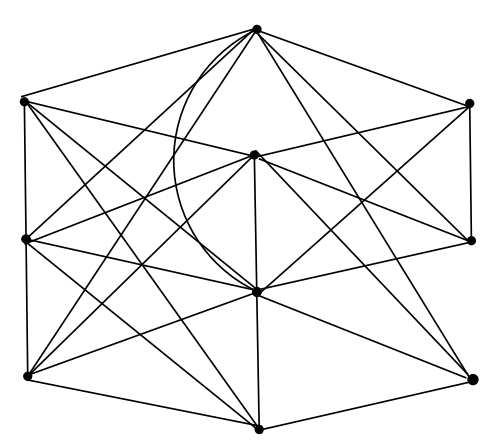

We see that $\phi_{1}(\lambda)=\lambda^{3}-2 \lambda, \phi_{2}(\lambda)=\lambda^{4}-3 \lambda^{2}, \phi_{3}(\lambda)=\lambda^{3}-\lambda, \Gamma_{1}(\lambda)=\frac{3 \lambda+4}{\lambda^{2}-2}$, $\Gamma_{2}(\lambda)=\frac{4 \lambda+6}{\lambda^{2}-3}$ and $\Gamma_{3}(\lambda)=\frac{3 \lambda-1}{\lambda^{2}-\lambda}$. The characteristic polynomial of $G$ is $\lambda^{3}\left(\lambda^{3}+\right.$ $\left.4 \lambda^{2}-\lambda-6\right)\left(\lambda^{3}-5 \lambda^{2}-8 \lambda+2\right)(\lambda+1)$ which is equal to 


$$
\phi_{1}(\lambda) \phi_{2}(\lambda) \phi_{3}(\lambda) \Gamma_{1}(\lambda) \Gamma_{2}(\lambda) \Gamma_{3}(\lambda) \operatorname{det}\left[\begin{array}{ccc}
\frac{1}{\Gamma_{1}(\lambda)} & -1 & 0 \\
-1 & \frac{1}{\Gamma_{2}(\lambda)} & -1 \\
0 & -1 & \frac{1}{\Gamma_{3}(\lambda)}
\end{array}\right] \text {. }
$$

Example 2. Consider $H$ and $\mathcal{F}$ as in Example 1. Let $S_{1}=\left\{v_{1}^{(1)}, v_{2}^{(1)}\right\}, S_{2}=$ $\left\{v_{1}^{(2)}, v_{2}^{(2)}, v_{4}^{(2)}\right\}$ and $S_{3}=\left\{v_{2}^{(3)}, v_{3}^{(3)}\right\}$. Then the $H$-generalized join graph $G=\bigvee_{H, S} \mathcal{F}$ is given as

$G$ :

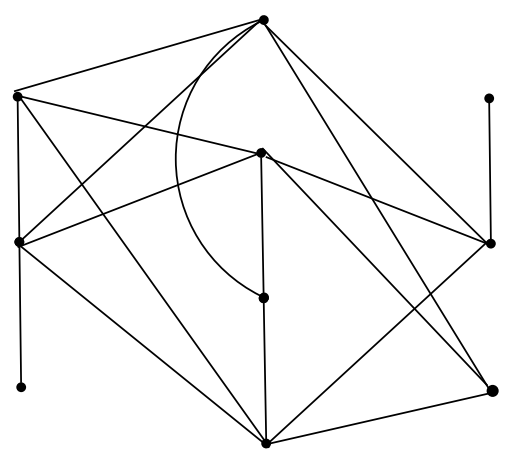

Here, $\phi_{1}(\lambda)=\lambda^{3}-2 \lambda, \phi_{2}(\lambda)=\lambda^{4}-3 \lambda^{2}$ and $\phi_{3}(\lambda)=\lambda^{3}-\lambda$. Based on the choices of $S_{1}, S_{2}$ and $S_{3}$ we get $\Gamma_{1}\left(\chi_{S_{1}} ; \lambda\right)=\frac{2 \lambda^{2}+2 \lambda-1}{\lambda^{3}-2 \lambda}, \Gamma_{2}\left(\chi_{S_{2}} ; \lambda\right)=\frac{3 \lambda}{\lambda^{2}-3}$ and $\Gamma_{3}\left(\chi_{S_{3}} ; \lambda\right)=$ $\frac{2 \lambda^{2}-1}{\lambda^{3}-\lambda}$. The characteristic polynomial of $G$ is $\lambda^{4}\left(\lambda^{6}-18 \lambda^{4}-6 \lambda^{3}+35 \lambda^{2}+6 \lambda-15\right)$ which is equal to

$\phi_{1}(\lambda) \phi_{2}(\lambda) \phi_{3}(\lambda) \Gamma_{1}\left(\chi_{S_{1}} ; \lambda\right) \Gamma_{2}\left(\chi_{S_{2}} ; \lambda\right) \Gamma_{3}\left(\chi_{S_{3}} ; \lambda\right) \operatorname{det}\left[\begin{array}{ccc}\frac{1}{\Gamma_{1}\left(\chi_{S_{1}} ; \lambda\right)} & -1 & 0 \\ -1 & \frac{1}{\Gamma_{2}\left(\chi_{S_{2}} ; \lambda\right)} & -1 \\ 0 & -1 & \frac{1}{\Gamma_{3}\left(\chi_{S_{3}} ; \lambda\right)}\end{array}\right]$.

\section{Declaration of competing interest}

There is no competing interest.

\section{References}

[1] M.S. Bartlett, An inverse matrix adjustment arising in discriminant analysis, Ann. Math. Stat. 22 (1951) 107-111.

[2] S. Butler, A note about cospectral graphs for the adjacency and normalized Laplacian matrices, Linear Multilinear Algebra 58 (2010) 387-390.

[3] D.M. Cardoso, I. Gutman, E.A. Martins, M. Robbiano, A generalization of Fiedler's lemma and some applications, Linear Multilinear Algebra 59 (8) (2011) 929-942. 
[4] D.M. Cardoso, M.A. de Freitas, E.A. Martins, M. Robbiano, Spectra of graphs obtained by a generalization of the join graph operation, Discrete Math. 313 (2013) 733-741.

[5] D.M. Cardoso, E.A. Martins, M. Robbiano, O. Rojo, Eigenvalues of a H-generalized join graph operation constrained by vertex subsets, Linear Algebra Appl. 438 (8) (2013) 3278-3290.

[6] D.M. Cardoso, P. Rama, Spectral results on regular graphs with (k,tau)-regular sets, Discrete Math. 307 (2007) 1306-1316.

[7] D.M. Cardoso, I. Sciriha, C. Zerafa, Main eigenvalues and $(k, \tau)$-regular sets, Linear Algebra Appl. 432 (2010) 2399-2408.

[8] Y. Chen, H. Chen, The characteristic polynomial of a generalized join graph, Appl. Math. Comput. 348 (2019) 456-464.

[9] D. Cvetković, M. Doob, H. Sachs, Spectra of Graphs: Theory and Application, Johann Ambrosius Barth Verlag, Heidelberg-Leipzig, 1995.

[10] D. Cvetković, P. Rowlinson, S. Simić, An Introduction to the Theory of Graph Spectra, Cambridge University Press, 2010.

[11] J. Ding, A. Zhou, Eigenvalues of rank-one updated matrices with some applications, Appl. Math. Lett. 20 (2007) 1223-1226.

[12] M. Fiedler, Eigenvalues of nonnegative symmetric matrices, Linear Algebra Appl. 9 (1974) 119-142.

[13] A.R.F. Laali, H.H. Seyyedavadi, D. Kiani, Spectra of generalized corona of graphs, Linear Algebra Appl. 493 (2016) 411-425.

[14] A. Gerbaud, Spectra of generalized compositions of graphs and hierarchical networks, Discrete Math. 310 (2010) 2824-2830.

[15] C. Godsil, G. Royle, Algebraic Graph Theory, Springer-Verlag, New York, 2001.

[16] W.H. Haemers, M.R. Oboudi, Universal spectra of the disjoint union of regular graphs, Linear Algebra Appl. 606 (2020) 244-248.

[17] C. McLeman, E. McNicholas, Spectra of coronae, Linear Algebra Appl. 435 (2011) 998-1007.

[18] A. Neumaier, Regular sets and quasi-symmetric 2-designs, in: D. Jungnickel, K. Vedder (Eds.), Combinatorial Theory, Springer, Berlin, 1982, pp. 258-275.

[19] M. Neumann, S. Pati, The Laplacian spectra with a tree structure, Linear Multilinear Algebra 57 (3) (2009) 267-291.

[20] P. Rowlinson, The main eigenvalues of a graph: a survey, Appl. Anal. Discrete Math. 1 (2007) 445-471.

[21] A.J. Schwenk, Computing the characteristic polynomial of a graph, in: R. Bary, F. Harary (Eds.), Graphs Combinatorics, in: Lecture Notes in Mathematics, vol. 406, Springer-Verlag, Berlin, 1974, pp. $153-172$.

[22] D. Stevanović, Large sets of long distance equienergetic graphs, Ars Math. Contemp. 2 (2009) 35-40.

[23] Z. Wang, D. Wong, The characteristic polynomial of lexicographic product of graphs, Linear Algebra Appl. 541 (2018) 177-184.

[24] B.-F. Wu, Y.-Y. Lou, C.-X. He, Signless Laplacian and normalized Laplacian on the H-join operation of graphs, Discrete Math. Algorithms Appl. 6 (3) (2014) 1450046-1-1450046-13. 